\title{
Mathematical modeling of forest ecosystems by a reaction-diffusion-advection system: impacts of climate change and deforestation
}

\author{
Guillaume Cantin • Arnaud Ducrot • \\ Beatriz M. Funatsu
}

2021, November 18

\begin{abstract}
We present an innovative mathematical model for studying the dynamics of forest ecosystems. Our model is determined by an age-structured reaction-diffusion-advection system in which the roles of the water resource and of the atmospheric activity are considered. The model is abstract but constructed in such a manner that it can be applied to real-world forest areas; thus it allows to establish an infinite number of scenarios for testing the robustness and resilience of forest ecosystems to anthropic actions or to climate change. We establish the well-posedness of the reaction-diffusion-advection model by using the method of characteristics and by reducing the initial system to a reaction-diffusion problem. The existence and stability of stationary homogeneous and stationary heterogeneous solutions are investigated, so as to prove that the model is able to reproduce relevant equilibrium states of the forest ecosystem. We show that the model fits with the principle of almost uniform precipitation over forested areas and of exponential decrease of precipitation over deforested areas. Furthermore, we present a selection of numerical simulations for an abstract forest ecosystem, in order to analyze the stability of the steady states, to investigate the impact of anthropic perturbations such as deforestation and to explore the effects of climate change on the dynamics of the forest ecosystem.
\end{abstract}

Keywords Forest model · Water resource · Climate change · Reactiondiffusion-advection · Stability analysis.

2020 Mathematics Subject Classification. 92B05; 35K58; 35K65; 35P15.

G. Cantin, Laboratoire des Sciences du Numérique, LS2N UMR CNRS 6004, Université de Nantes, France. E-mail: guillaume.cantin@univ-nantes.fr

A. Ducrot, Laboratoire de Mathématiques Appliquées, FR CNRS 3335, Université Le Havre Normandie, France. E-mail: arnaud.ducrot@univ-lehavre.fr B.M. Funatsu, CNRS, UMR 6554 LETG, Batiment IGARUN, Campus du Tertre, Université de Nantes, France. E-mail: beatriz.funatsu@univ-nantes.fr 


\section{Introduction}

Over the past few years, scientific research has paid particular attention to the nexus between the equilibrium of forest ecosystems and climate change, in an international context of global warming which threatens the health of biodiversity as well as the running and progress of our societies. A huge literature has emerged on this topic thus it is not reasonable to expect an exhaustive review of the related papers. One can however mention several significant studies dedicated to forest management [8], [17], [26], [32], [34], forest fires [12], [13], [25], [29], [44], [41], or to the role of forest ecosystems on carbon fixation [6], [42], [45]. Recently, the impact of deforestation on the climate change has encountered a growing interest [1], [14], [36], and it is now well admitted that the protection of forest ecosystems will represent a major challenge in the near future.

In this scientific landscape, mathematical modeling plays a crucial role for a better understanding and predicting of the dynamics of forest ecosystems. An inescapable work on this subject is certainly the book of Botkin [3], where the ecological mechanisms which occur within forest ecosystems are reviewed, so as to prepare the research of realistic models. Pioneer works on the modeling of forest ecosystems by dynamical systems and differential equations can be found in [2], where the age-structure of the forest is considered, or in [18], where the multi-species distribution of trees is taken into account. Age or sizestructured models are also studied in [20] and [23], where partial differential equations such as reaction-diffusion equations or cross-diffusion equations are introduced. In parallel, agent-based models have also been studied, for example in [19] or [37], with a numerical approach. Besides competition and cooperation mechanisms which take place within the population of trees composing the forest ecosystem, it is essential to analyze the effect of exterior parameters such as the water resource and the atmospheric activity, since extreme events (e.g. droughts or storms) obviously exert a high pressure on forests. In a recent paper [4], complex networks of forest patches have been studied with the aim to model the interaction between two given forest patches through water exchanges; in this work, the water exchanges have been modeled by considering the forest evapotranspiration phenomenon, which is well understood for several decades as a key factor of the water cycle [7], [28], [39]. It is worth noting that the results obtained in [4] by numerical simulations of a complex network of differential equations converge with the observations discussed in [43], where self-amplified forest loss due to deforestation or under dry-season intensification is investigated for the Amazon forest. These recent works represent original attempts to integrate the role of the water resource on the dynamics of forest ecosystems. However, the modeling of tree ages, tree densities, their spatial distributions and their contribution to evapotranspiration, has not been explicitly addressed in the models aforementioned. It is precisely the original contribution of the present work to fill these lacks.

Therefore, we present in this article an innovative mathematical model for studying the dynamics of forest ecosystems and their nexus with the wa- 
ter resource and the atmospheric activity. Stemming from a spatio-temporal age-structured model presented in [20] and analyzed with a theoretical approach in [21], [22], we integrate firstly the effects of the atmospheric moisture transport by supplementing the original model with a moisture conservation equation, and secondly the role of the water resource by considering biological parameters of the forest as functions of the precipitation quantity. In particular, we assume that a lack of the water resource causes an increase of the mortality rates of trees and a decrease of the seeds production. In parallel, we assume that a high density of trees in the forest favors the evapotranspiration mechanism, whereas a weak density of trees induces an attenuation of evapotranspiration. The moisture transport is modeled by an advection field which is assumed to emerge over oceanic areas and to carry the water resource across the whole continental area. In our work, we choose to focus on the time evolution of the forest ecosystem, thus processes which act at a relatively fast time scale, such as the wind activity, shall be subject to approximations, whereas processes which act at a relatively slow time scale, such as climate change, shall be treated by a change of parameters. Our modeling approach leads to a reaction-diffusion-advection system; we establish the well-posedness of this reaction-diffusion-advection model in a rigorous mathematical framework, by using the method of characteristics and by reducing the initial system to a reaction-diffusion problem. Next, we validate our model by proving that it is in agreement with the principle of almost uniform precipitation over forested areas and of exponential decrease of precipitation over deforested areas. Our model is abstract, however we emphasize that it is constructed in such a manner that it can be adapted to various real-world forest areas, such as the Amazon forest, the Congo basin forest or the Siberian forest. Thus the model allows to establish an infinite number of scenarios for testing a perturbation of the atmospheric activity, which can be caused by climate change, as well as anthropic actions such as deforestation. For example, the possible scenario of a drought can be experimented, since the parameters of the model vary with respect to the water resource quantity. Here, we explore the existence of stationary homogeneous and stationary heterogeneous solutions, and analyze their stability. We prove that the model admits a stationary heterogeneous solution modeling a desert area; we search for conditions of instability of that particular solution, which can be viewed as the possibility of regenerateness of the forest, whereas stability corresponds to the impossibility of recreating a healthy forest. We also prove that the model admits stationary homogeneous solutions which model good health equilibrium states of the forest. Finally, we present a selection of relevant numerical simulations which are performed in order to test the effect of climate change on the equilibrium of the forest and the resilience of the ecosystem to various strategies of deforestation.

Our paper is organized as follows. In the next section, we show how our model is constructed, by improving a given age-structured reaction-diffusion system and by incorporating the effects of the atmospheric moisture transport and of the water resource. Afterwards, we present the mathematical analysis of the reaction-diffusion-advection model and explore the existence and stability 
of equilibrium states for the water-forest system. To that aim, we establish the existence of several stationary solutions; some of these solutions are uniform in space and time, whereas other are uniform in time but not in space. In the final section, we show that our model reproduces basic patterns of spatial distribution of precipitation, which represents a first step in the validation of the model. Then we focus on the stability of a particular stationary solution by a numerical approach, so as to experiment the impacts of climate change and deforestation.

\section{Presentation of the model}

In this first section, we present our innovative mathematical model, which is designed in order to study the dynamics of forest ecosystems and their nexus with the water resource. The mathematical model takes the form of an evolution problem in which advection and reaction-diffusion processes are coupled.

2.1 Modeling the water resource spatial distribution

Let us consider a bounded domain $\Omega \subset \mathbb{R}^{2}$ with regular boundary $\partial \Omega$, which models a geographical region occupied by a forest ecosystem.

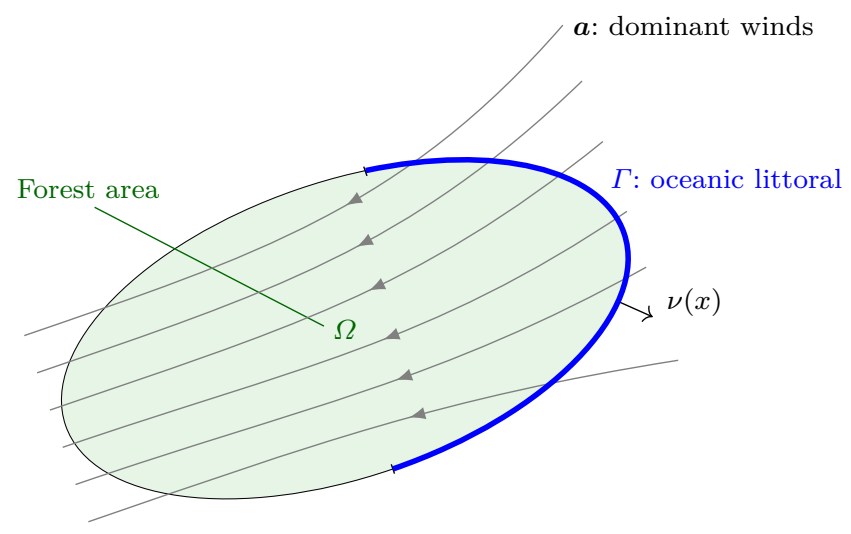

Fig. 1 Bounded domain $\Omega$ modeling a geographical area occupied by a forest. The domain is crossed by an advection field $\boldsymbol{a}$ modeling the dominant winds, whose characteristic lines enter by the border $\Gamma$, which models the oceanic littoral.

We denote by $x=\left(x_{1}, x_{2}\right) \in \mathbb{R}^{2}$ the space variable and by $t$ the time variable. We assume that the scale of the time variable $t$ describes the evolution of the forest ecosystem; in contrast, the evolution of the dominant winds is described at a fast scale by a second time variable $\tau$ (see Remark 1 for details on the precipitation and vegetation timescales in the example of the Amazon 
region). In our work, as mentioned above, we choose to focus on the time evolution of the forest ecosystem, thus the processes which act at the fast time scale $\tau$ shall be subject to averaging.

As illustrated in figure 1, we assume that the domain $\Omega$ is crossed by an advection field $\boldsymbol{a}=\boldsymbol{a}(x)$ reproducing the per year averaged activity of dominant winds, and we assume this time average does not vary over the years. We also assume that the characteristic lines of the advection field $\boldsymbol{a}$ enter the domain $\Omega$ by the border $\Gamma$, which models the oceanic littoral, defined by

$$
\Gamma=\{x \in \partial \Omega ; \boldsymbol{a}(x) \cdot \nu(x)<0\},
$$

where $\nu(x)$ denotes the outward normal vector at point $x \in \partial \Omega$. As an example, the domain $\Omega$ can model the Amazon forest, in which case the border $\Gamma$ corresponds to the Atlantic littoral. The advection field $\boldsymbol{a}$ can be perturbed by various processes such as climate changes, which act at a very long time scale which is not commensurate with the forest ecosystem time scale $t$; rather than integrating the very long time scale in our model, we shall treat these perturbations of the advection field $\boldsymbol{a}$ by a change of parameters.

We denote by $\rho(t, x)$ the water quantity per unit of surface, by $u(t, x)$ the surface density of young trees, by $v(t, x)$ the surface density of old trees and by $w(t, x)$ the surface density of seeds for each $t \geq 0$ and each $x \in \Omega$. We assume that the water resource has two distinct origins. The first contribution is stemming from climatic evaporation over maritime zones, whereas the second contribution is the result of the evapotranspiration over forest. We propose to model the distribution of the per year averaged water quantity by a moisture conservation equation which reads

$$
\frac{\partial \rho}{\partial t}+\boldsymbol{a} \cdot \nabla \rho=-\sigma \rho+\varphi(\rho) v
$$

Considering that the per year average water kinematics are much more rapid than the forest kinematics for adult trees (several years), it is reasonable to assume that the solution of the non-stationary equation (1), between two relevant time steps of the forest dynamics, evolves rapidly towards a stationary solution (we show in the Appendix numerical results which underpin this approximation). We emphasize that this fast convergence has been proved in a rigorous mathematical framework for a wide class of nonlinear transport equations, for instance in [30]. Thus we assume that the water resource distribution can be approximated by the following stationary advection equation

$$
\boldsymbol{a} \cdot \nabla \rho(t, x)=-\sigma \rho(t, x)+\varphi(\rho(t, x)) v(t, x) .
$$

Here the parameter $\sigma$ corresponds to the per year averaged decay rate of the water resource evaporated over the maritime zone or over the forest zone, when it is brought inland by the atmospheric advection (the rate $\sigma$ can be determined by considering the probability for a given water molecule standing in atmospheric moisture to precipitate on soil). The perturbations of $\sigma$ by very long time processes such as climate change, shall be treated by a change of 
parameters, as for the advection field $\boldsymbol{a}$. The forest evapotranspiration process is modeled by the term $\varphi(\rho) v$, where $\varphi$ is a non-negative increasing function defined for $v \geq 0$ and such that

$$
\varphi(0)=0, \quad \lim _{\rho \rightarrow+\infty} \varphi(\rho)=\varphi_{0}>0
$$

The latter limit models a saturation process: when the available water resource overcomes a given threshold, then the evapotranspiration process does not vary any more. The shape of function $\varphi$ is illustrated in figure 3 (a). With this modeling choice, we consider that young trees do not contribute to forest evapotranspiration, and that the forest evapotranspiration is vanishing when the water resource decreases. Note that the water quantity $\rho$ still depends on the slow time variable $t$, since the density of old trees $v$ varies with $t$. We supplement the stationary advection equation (2) by a partial boundary condition defined on $\Gamma$ :

$$
\rho(t, x)=m(x), \quad x \in \Gamma
$$

where $m$ is a regular function modeling the per year averaged oceanic contribution to the water cycle. Here again, we assume this average does not vary in time while slow variations due to climate changes for instance can be handled by parameter changes. In the sequel of the paper, we shall make several assumptions on the vector field $\boldsymbol{a}$ and on the set $\Gamma$, in order to integrate the advection equation (2) using the method of characteristics (see equations (14)-(15)-(16)-(17) in subsection 3.1).

Remark 1 (Example of the Amazon region) Climate in the Amazon region presents a marked seasonality, except for the mean air temperature, which presents a small annual amplitude in most (albeit not all) of the region. Precipitation seasonality has strong spatial variations with short or absent dry season in the west and northwest, in contrast to a very seasonal regime in the south and southeast with longer dry seasons and a relatively rapid transition between the wet and dry season (see e.g. [9] or [24]). Precipitation patterns in the Amazon basin are, in turn, strongly linked to inter-annual and interdecadal tropical sea surface temperatures in both Pacific and Atlantic oceans (see e.g. [10]). In terms of climate-vegetation equilibrium timescales, Oyama \& Nobre [27] performed climate simulations using an atmospheric general circulation model to examine the potential equilibrium states after a complete Earth deforestation scenario, and found that a simulation run of 15 years (with vegetation updated every 3 years) was sufficient for the climate-vegetation system to reach equilibrium (notice that oceanic and atmospheric parameters were updated at monthly scale in their model). This climate-vegetation equilibrium timescale (10 years) seems reasonable, as the climatic response time of the vegetation is expected to be small compared to the time scale of climatic variation to which it is responding [38]. 
2.2 Dynamics of the water-forest ecosystem

Next, we incorporate the advection equation (2) into the forest kinematics model proposed by [20]. Thus we consider the following reaction-diffusionadvection problem:

$$
\left\{\begin{aligned}
\boldsymbol{a} \cdot \nabla \rho & =-\sigma \rho+\varphi(\rho) v \\
\frac{\partial u}{\partial t} & =\beta \delta w-\gamma(v, \rho) u-f u \\
\frac{\partial v}{\partial t} & =f u-h(\rho) v \\
\frac{\partial w}{\partial t} & =d \Delta w-\beta w+\alpha(\rho) v
\end{aligned}\right.
$$

We recall that the fast dynamic of atmospheric activity with respect to the slow dynamic of forest evolution justifies the assumption of a stationary advection equation for the distribution of the water resource. These equations are supplemented by the partial boundary condition (4) on $\rho$ and the Neumann boundary condition on $w$ :

$$
\frac{\partial w}{\partial \nu}(t, x)=0, t \geq 0, x \in \partial \Omega,
$$

which models the obvious impossibility for the forest to colonize the exterior of the domain $\Omega$, and the possible accumulation of seeds along the continental border (however, note that a forest model with Dirichlet boundary condition has been studied in [31]). Finally, initial conditions are given by:

$$
u(0, x)=u_{0}(x), v(0, x)=v_{0}(x), w(0, x)=w_{0}(x), x \in \Omega,
$$

where $u_{0}, v_{0}$ and $w_{0}$ are smooth non-negative functions defined in $\Omega$.

The unknowns and parameters of our model are gathered in table 1 , and the dynamics of system (5) are illustrated in figure 2 . In our model, we assume that the spatial distribution of the water resource $\rho(t, x)$ affects the dynamics of the forest: for that reason, the mortality rates $\mu(\rho), h(\rho)$ of young and old trees respectively, as well as the production rate of seeds $\alpha(\rho)$, will depend on $\rho$. More precisely, the global mortality rate of young trees is given by $\gamma(\rho, v)$. We assume that this mortality rate can be written into the separated form

$$
\gamma(\rho, v)=\gamma_{0}(v)+\mu(\rho) .
$$

Here, the function $\gamma_{0}$ models the competition with old trees; we assume that $\gamma_{0}$ is given by a polynomial expression of the form

$$
\gamma_{0}(v)=a(v-b)^{2}+c
$$

with positive coefficients $a, b, c$. This quadratic expression models the competition between young and old trees; it is assumed that there exists an optimal 
Table 1 Unknowns and parameters of the forest model (5).

\begin{tabular}{lll}
\hline Unknown & Symbol & Unit \\
\hline Water resource & $\rho(t, x)$ & $\mathrm{mm} \times \mathrm{ha}^{-1}$ \\
Young trees density & $u(t, x)$ & trees $\times \mathrm{ha}^{-1}$ \\
Old trees density & $v(t, x)$ & trees $\times \mathrm{ha}^{-1}$ \\
Seeds density & $w(t, x)$ & seeds $\times \mathrm{ha}^{-1}$ \\
\hline Parameter & Symbol & $\mathrm{Unit}^{-1}$ \\
\hline Oceanic contribution to water resource & $m(x)$ & $\mathrm{mm} \times \mathrm{ha}^{-1}$ \\
Maximum water production rate & $\alpha_{0}$ & $\mathrm{yr}^{-1}$ \\
Maximum mortality rate of young trees & $\mu_{0}$ & $\mathrm{yr}^{-1}$ \\
Seeds production rate & $\beta$ & $\mathrm{yr}^{-1}$ \\
Seeds establishment rate & $\delta$ & $\mathrm{yr}^{-1}$ \\
Aging rate & $f$ & $\mathrm{yr}^{-1}$ \\
Maximum mortality rate of old trees & $h_{0}$ & $\mathrm{yr}^{-1}$ \\
Minimum mortality rate of old trees & $h_{1}$ & $\mathrm{yr}^{-1}$ \\
Decreasing rate of water resource & $\sigma$ & $\mathrm{yr}^{-1}$ \\
Maximum contribution rate to evaporation & $\varphi_{0}$ & $\mathrm{~mm} \times \mathrm{trees}^{-1} \times \mathrm{yr}^{-1}$ \\
Parameters of competition & $a$ & $\mathrm{trees}^{-2} \times \mathrm{ha}^{2} \times \mathrm{yr}^{-1}$ \\
between young and old trees & $b$ & $\mathrm{trees}^{-1} \mathrm{ha}^{-1}$ \\
Diffusion rate of seeds & $c$ & $\mathrm{yr}^{-1}$ \\
\hline
\end{tabular}

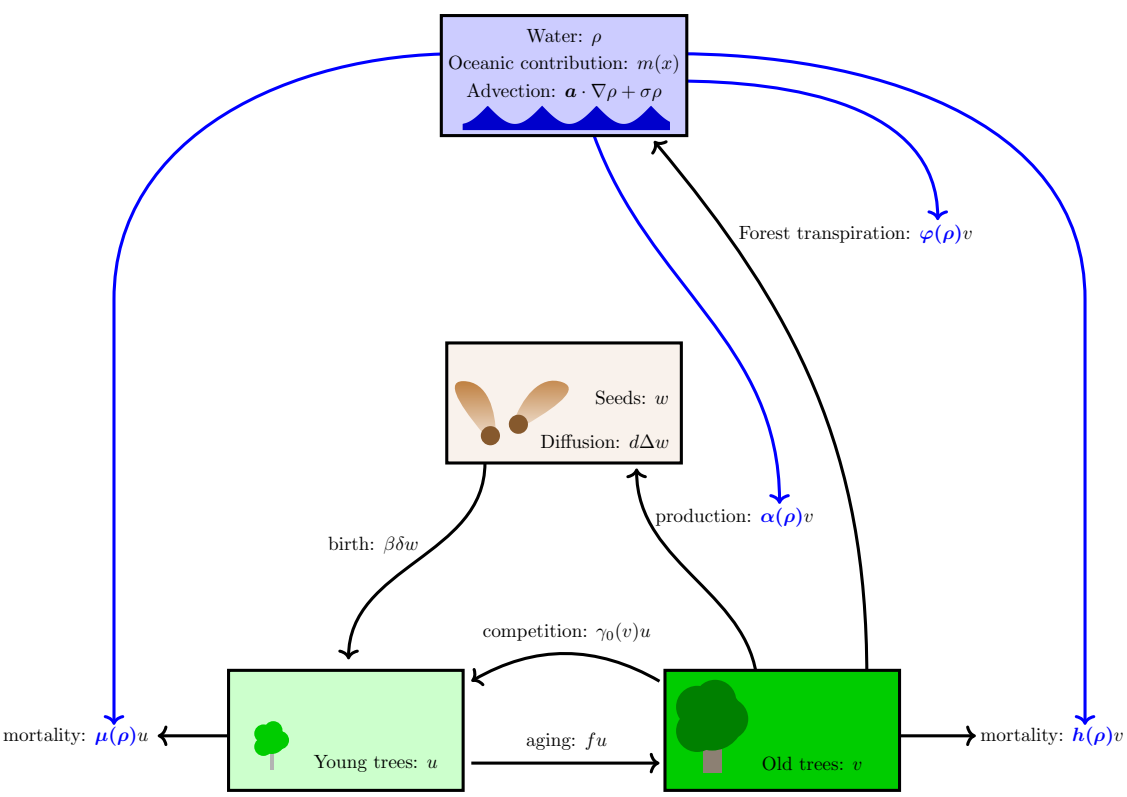

Fig. 2 Graphical model illustrating the dynamics of a water-forest ecosystem. We assume that the spatial distribution of the water resource affects the mortalities rates $\mu(\rho)$ and $h(\rho)$ of young and old trees respectively, as well as the forest transpiration rate $\varphi(\rho)$ and the production rate of seeds $\alpha(\rho)$. 


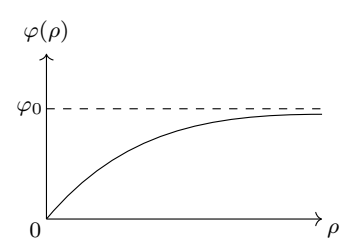

(a)

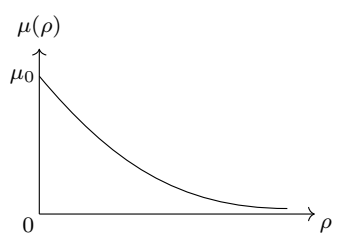

(b)

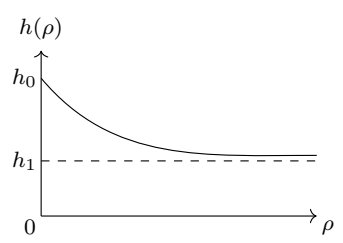

(c)



(d)

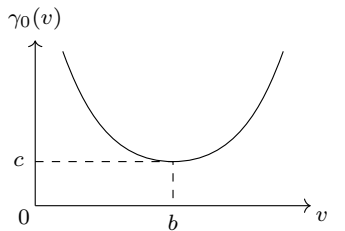

(e)

Fig. 3 Graphs of functions $\varphi(\rho), \mu(\rho), h(\rho), \alpha(\rho), \gamma_{0}(v)$ involved in model (5). (a) The function $\varphi(\rho)$ models the contribution of old trees to water evaporation over forest, depending on the water resource $\rho$. (b), (c) The functions $\mu(\rho)$ and $h(\rho)$ model the mortalities of young and old trees respectively, with respect to a variation of the water resource. (d) The function $\alpha(\rho)$ models the production of seeds by old trees. (e) The function $\gamma_{0}(v)$ models the mortality of young trees due to the competition with old trees.

configuration, corresponding to the minimum of the quadratic function $\gamma_{0}$, where the competition is attenuated. Indeed, in total absence of old trees, young trees could be exposed to bad weather and present an increase of mortality; but in overabundance of old trees, young trees could suffer a lack of water and light resources. The function $\mu(\rho)$ models the effect of the water resource on the mortality rate of young trees. We assume that the over-mortality rate $\mu$ is a positive decreasing function such that

$$
\mu(0)=\mu_{0}>0, \quad \lim _{\rho \rightarrow+\infty} \mu(\rho)=0 .
$$

The latter limit models again a saturation process: when the available water resource overcomes a given threshold, then the mortality rate does not vary any more. However, a lack of water resource increases the mortality of young trees. The graphs of functions $\gamma_{0}$ and $\mu$ are illustrated in figure 3 (e) and (b) respectively.

Next, the mortality rate of old trees is given by $h(\rho)$. We assume, as illustrated in figure 3 (c), that $h$ is a positive decreasing function of $\rho$ such that

$$
h(0)=h_{0}>0, \quad \lim _{\rho \rightarrow+\infty} h(\rho)=h_{1}>0 .
$$

As for young trees, a lack of water resource increases the mortality of old trees, but the mortality stabilizes if the available water resource overcomes a given saturation threshold.

Furthermore, the aging rate of young trees is a positive coefficient denoted by $f$. The evolution of seeds density is described by fourth equation in system 
(5). This evolution admits a production by old trees term given by $\alpha(\rho) v$, where the function $\alpha$ satisfies similar properties as function $\varphi$, that is

$$
\alpha(0)=0, \quad \lim _{\rho \rightarrow+\infty} \alpha(\rho)=\alpha_{0}>0
$$

The germination process of seeds is modeled by the term $\beta \delta w$ in which $\beta$ and $\delta$ are positive coefficients modeling the seed production rate and the seed establishment rate respectively. Finally, the evolution of seeds density admits a diffusion in the air effect, modeled by the Laplace operator weighted by the positive diffusion coefficient $d$.

\section{Well-posedness and global solutions of the reaction-diffusion-advection system}

In this section, we prove that system (5) admits global in time solutions with non-negative and bounded components $(\rho, u, v, w)$, for any non-negative and sufficiently smooth initial conditions $u_{0}, v_{0}$ and $w_{0}$ defined in $\Omega$. Note that some of the solutions may admit discontinuities, as established in [22] for the model without water resource.

\subsection{Reduction to a reaction-diffusion system}

Here and in the rest of the paper, we assume that the functions $\alpha, \varphi, \mu, h$ are continuously differentiable real-valued functions satisfying the following properties:

$$
\begin{array}{ll}
0 \leq \alpha(s) \leq \alpha_{0}, & \left|\alpha\left(s_{1}\right)-\alpha\left(s_{2}\right)\right| \leq \alpha_{0}\left|s_{1}-s_{2}\right| \\
0 \leq \varphi(s) \leq \varphi_{0}, & \left|\varphi\left(s_{1}\right)-\varphi\left(s_{2}\right)\right| \leq \varphi_{0}\left|s_{1}-s_{2}\right| \\
0 \leq \mu(s) \leq \mu_{0}, & \left|\mu\left(s_{1}\right)-\mu\left(s_{2}\right)\right| \leq \mu_{0}\left|s_{1}-s_{2}\right| \\
h_{1} \leq h(s) \leq h_{0}, & \left|h\left(s_{1}\right)-h\left(s_{2}\right)\right| \leq h_{0}\left|s_{1}-s_{2}\right|
\end{array}
$$

for all $s, s_{1}, s_{2} \in \mathbb{R}^{+}$, with positive coefficients $\alpha_{0}, \varphi_{0}, \mu_{0}, h_{0}$ and $h_{1}$ (such that $\left.h_{1}<h_{0}\right)$. Explicit expressions are used in the last section of our paper, to perform numerical simulations (see equations (42)). The function $\gamma_{0}$ is defined as in [20] by (9). We also make assumptions on the advection field $\boldsymbol{a}=\left(a_{1}, a_{2}\right)$ : we assume the characteristic lines of $\boldsymbol{a}$, determined by the orbits of the differential system

$$
\left\{\begin{array}{l}
\frac{d x_{1}}{d s}=a_{1}\left(x_{1}, x_{2}\right) \\
\frac{d x_{2}}{d s}=a_{2}\left(x_{1}, x_{2}\right),
\end{array}\right.
$$


with $x(0)=x_{0} \in \Gamma$, cover the domain $\Omega$ in such a way that each point $x \in \Omega$ belongs to a unique characteristic line. We denote by $\left\{\xi\left(x_{0}, s\right)\right\}_{0 \leq s \leq S\left(x_{0}\right)}$ the unique orbit of system (14) stemming from $x_{0} \in \Gamma$. We assume that

$$
\xi\left(x_{0}, 0\right)=x_{0}, \quad \xi\left(x_{0}, s\right) \in \Omega \text { if } 0<s<S\left(x_{0}\right), \quad \xi\left(x_{0}, S\left(x_{0}\right)\right) \in \partial \Omega \backslash \Gamma,
$$

and that $\boldsymbol{a}$ is sufficiently smooth, so that the family of orbits $\left\{\xi\left(x_{0}, s\right)\right\}_{0 \leq s \leq S\left(x_{0}\right)}$ parametrized by $x_{0} \in \Gamma$ vary continuously with $x_{0}$, that is

$$
\sup _{0 \leq s \leq \min \left(S\left(x_{0}\right), S\left(x_{1}\right)\right)}\left\|\xi\left(x_{0}, s\right)-\xi\left(x_{1}, s\right)\right\|_{\mathbb{R}^{2}} \rightarrow 0
$$

as $\left\|x_{0}-x_{1}\right\|_{\mathbb{R}^{2}}$ tends to 0 . Furthermore, we assume that there exists a positive constant $\bar{S}$ such that

$$
S\left(x_{0}\right) \leq \bar{S}, \quad \forall x_{0} \in \Gamma
$$

Note that the latter assumption is directly satisfied if $\Gamma$ is compact.

Next, for each $x \in \Omega$, we denote the unique pair $\left(x_{0}, s\right) \in \Gamma \times \mathbb{R}$ such that $x=\xi\left(x_{0}, s\right)$ by

$$
\left(x_{0}, s\right)=\left(\zeta_{1}(x), \zeta_{2}(x)\right) .
$$

With the latter notation, the unique orbit of system (14) passing through $x \in \Omega$ is $\left\{\xi\left(\zeta_{1}(x), s\right)\right\}_{0 \leq s \leq S\left(\zeta_{1}(x)\right)}$. The continuity of $\boldsymbol{a}$ implies that of $\zeta_{1}$ and $\zeta_{2}$.

Notation. Let $q$ denote a function defined almost everywhere in $\Omega$. We have

$$
q(x)=q\left(\xi\left(\zeta_{1}(x), \zeta_{2}(x)\right)\right),
$$

for $x$ almost everywhere in $\Omega$. We introduce the notation

$$
\tilde{q}\left(x_{0}, s\right)=q \circ \xi\left(x_{0}, s\right), \quad x_{0} \in \Gamma, \quad s \in\left[0, S\left(x_{0}\right)\right] .
$$

In order to lighten the latter notation, and if the context is sufficiently clear, we may simply write $\tilde{q}(s)$ instead of $\tilde{q}\left(x_{0}, s\right)$.

Now, for each $v \in L_{+}^{\infty}(\Omega)$ and each $m \in L_{+}^{\infty}(\Gamma)$, we consider the stationary advection equation

$$
\left\{\begin{array}{rlrl}
\boldsymbol{a} \cdot \nabla \rho & =-\sigma \rho+\varphi(\rho) v, & x \in \Omega, \\
\rho(x) & =m(x), & x & \in \Gamma,
\end{array}\right.
$$

where $L_{+}^{\infty}(\Omega)$ denotes the subset of $L^{\infty}(\Omega)$ with non-negative functions almost everywhere. Our aim is to study the following operator:

$$
\begin{aligned}
\psi: L_{+}^{\infty}(\Omega) & \longrightarrow L_{+}^{\infty}(\Omega) \\
v & \longmapsto \rho
\end{aligned}
$$

where $\rho$ is the solution of the advection equation (19). In the sequel, the norm of an element $u \in L^{\infty}(\Omega)$ will be denoted $\|u\|_{\infty}$. 
Theorem 1 The operator $\psi$ given by (20) is well defined and it is uniquely determined along the characteristic lines of the advection field $\boldsymbol{a}$ by

$$
\begin{aligned}
\psi(v)(x) & =m\left(\zeta_{1}(x)\right) e^{-\sigma \zeta_{2}(x)} \\
& +\int_{0}^{\zeta_{2}(x)} \varphi\left(\tilde{\rho}\left(\zeta_{1}(x), \tau\right)\right) \tilde{v}\left(\zeta_{1}(x), \tau\right) e^{-\sigma\left(\zeta_{2}(x)-\tau\right)} d \tau,
\end{aligned}
$$

for $x$ almost everywhere in $\Omega$ and for each $v \in L_{+}^{\infty}(\Omega)$, with the notation (18), where $\zeta_{1}$ and $\zeta_{2}$ are defined by (17).

Furthermore, the operator $\psi$ is continuous in $L_{+}^{\infty}(\Omega)$ and we have

$$
\|\psi(v+h)-\psi(v)\|_{\infty} \leq\|h\|_{\infty} \times \frac{\varphi_{0}}{\sigma} e^{\varphi_{0}\|v\|_{\infty} \bar{S}},
$$

for all $v, h$ in $L_{+}^{\infty}(\Omega)$. Finally, the operator $\psi$ is differentiable in $L_{+}^{\infty}(\Omega)$. Its derivative is given by

$$
\begin{aligned}
& D \psi(v) h(x)=\int_{0}^{\zeta_{2}(x)} \varphi\left(\tilde{\rho}\left(\zeta_{1}(x), \tau\right)\right) \tilde{h}\left(\zeta_{1}(x), \tau\right) e^{-\sigma\left(\zeta_{2}(x)-\tau\right)} d \tau \\
& +\int_{0}^{\zeta_{2}(x)} \varphi^{\prime}\left(\tilde{\rho}\left(\zeta_{1}(x), \tau\right)\right) \tilde{v}\left(\zeta_{1}(x), \tau\right) D \psi(v) \tilde{h}\left(\zeta_{1}(x), \tau\right) e^{-\sigma\left(\zeta_{2}(x)-\tau\right)} d \tau,
\end{aligned}
$$

for $x$ almost everywhere in $\Omega$ and for each $v, h \in L_{+}^{\infty}(\Omega)$, with the notation (18); its norm satisfies

$$
\|D \psi(v)\|_{\mathscr{L}\left(L^{\infty}(\Omega), L^{\infty}(\Omega)\right)} \leq \frac{\varphi_{0}}{\sigma} e^{\|v\|_{\infty} \bar{S}} .
$$

Proof The proof of Theorem 1 is given in the appendix.

3.2 Well-posedness of the reduced system

By virtue of Theorem 1, system (5) reduces to the following non-linear reactiondiffusion system:

$$
\begin{cases}\frac{\partial u}{\partial t}=\beta \delta w-\gamma(v, \psi(v)) u-f u, & t>0, x \in \Omega, \\ \frac{\partial v}{\partial t}=f u-h(\psi(v)) v, & t>0, x \in \Omega, \\ \frac{\partial w}{\partial t}=d \Delta w-\beta w+\alpha(\psi(v)) v, & t>0, x \in \Omega, \\ \frac{\partial w}{\partial \nu}(t, x)=0, & t \geq 0, x \in \partial \Omega .\end{cases}
$$

We consider the Banach space $X$ given by

$$
X=L^{\infty}(\Omega) \times L^{\infty}(\Omega) \times L^{2}(\Omega),
$$


and the linear operator $\Lambda$ determined by the realization of the operator $-d \Delta+$ $\beta$ in $L^{2}(\Omega)$ with the Neumann boundary condition on $\partial \Omega$. The norms in $L^{\infty}(\Omega), L^{2}(\Omega)$ and $X$ are denoted $\|\cdot\|_{\infty},\|\cdot\|_{2}$ and $\|\cdot\|_{X}$ respectively. We introduce the diagonal operator $A$ defined in $X$ by

$$
A=\operatorname{diag}\{f, 1, \Lambda\} .
$$

It is known that $A$ is a positive self-adjoint and sectorial operator in $X$, with angle strictly lesser than $\frac{\pi}{2}$, which generates an analytic semi-group and admits fractional powers. Its domain is given by

$$
\mathcal{D}(A)=L^{\infty}(\Omega) \times L^{\infty}(\Omega) \times H_{N}^{2}(\Omega),
$$

where $H_{N}^{2}(\Omega)=\left\{w \in H^{2}(\Omega) ; \frac{\partial w}{\partial \nu}=0\right.$ on $\left.\partial \Omega\right\}$. The domains of its fractional powers $A^{\eta}, 0<\eta<1$, are also well-known. We also introduce the non-linear operator $F$ defined by:

$$
F(U)=\left[\begin{array}{c}
\beta \delta w-\gamma(v, \psi(v)) u \\
f u-h(\psi(v)) v+v \\
\alpha(\psi(v)) v
\end{array}\right]=\left[\begin{array}{c}
F_{1}(U) \\
F_{2}(U) \\
F_{3}(U)
\end{array}\right]
$$

with $U=(u, v, w)^{T} \in \mathcal{D}\left(A^{\eta}\right)$, where $\eta$ is, now and in the rest of the paper, a fixed exponent such that $\frac{1}{2}<\eta<1$. Recall that by virtue of the Sobolev embeddings, we have

$$
\mathcal{D}\left(\Lambda^{\eta}\right)=H_{N}^{2 \eta}(\Omega) \subset \mathscr{C}(\bar{\Omega}),
$$

with continuous embedding. Consequently, we have $\mathcal{D}\left(A^{\eta}\right) \subset\left(L^{\infty}(\Omega)\right)^{3}$. We denote by $X_{0}$ the subset of $X$ whose elements are component-wise non-negative almost everywhere:

$$
X_{0}=\left\{U=(u, v, w)^{t} \in X ; u(x) \geq 0, v(x) \geq 0, w(x) \geq 0 \text { a.e. in } \Omega\right\} .
$$

The abstract formulation of system (25) reads:

$$
\left\{\begin{array}{l}
\frac{d U}{d t}+A U=F(U), \quad t>0 \\
U(0)=U_{0}
\end{array}\right.
$$

with $U_{0} \in X_{0}$.

Theorem 2 For any initial condition $U_{0} \in X_{0}$, the abstract Cauchy problem (27) admits a unique local in time solution $U=(u, v, w)^{T}$ defined on $\left[0, T_{U_{0}}\right]$, with

$$
\begin{aligned}
& u, v \in \mathscr{C}\left(\left[0, T_{U_{0}}\right], L^{\infty}(\Omega)\right) \cap \mathscr{C}^{1}\left(\left(0, T_{U_{0}}\right], L^{\infty}(\Omega)\right), \\
& w \in \mathscr{C}\left(\left(0, T_{U_{0}}\right], \mathcal{D}(\Lambda)\right) \cap \mathscr{C}\left(\left[0, T_{U_{0}}\right], L^{2}(\Omega)\right) \cap \mathscr{C}^{1}\left(\left(0, T_{U_{0}}\right], L^{2}(\Omega)\right),
\end{aligned}
$$


where $T_{U_{0}}>0$ depends on $\left\|U_{0}\right\|_{X}$ only. Furthermore, the local solution $U$ satisfies

$$
t\|A U(t)\|_{X}+\|U(t)\|_{X} \leq C_{U_{0}}, \quad 0<t \leq T_{U_{0}},
$$

where $C_{U_{0}}$ is a positive constant which depends only on $\left\|U_{0}\right\|_{X}$.

Proof The proof of Theorem 2 is also given in the appendix.

Next, we can prove that the local in time solutions of problem (25) starting in $X_{0}$ are non-negative component-wise, arguing as in [40] (Chapter 11, section 2.3). The association of Theorems 1 and 2 then leads to the following corollary.

Corollary 1 For any initial condition $U_{0} \in X_{0}$ and any non-negative function $m$ defined in $\Gamma$, the reaction-diffusion-advection problem (4)-(5)-(6)-(7) admits a unique local in time solution $(\rho, u, v, w)$ defined on $\left[0, T_{U_{0}}\right]$ with $T_{U_{0}}>0$ such that

$$
\begin{aligned}
& \rho, u, v \in \mathscr{C}\left(\left[0, T_{U_{0}}\right], L_{+}^{\infty}(\Omega)\right) \cap \mathscr{C}^{1}\left(\left(0, T_{U_{0}}\right], L_{+}^{\infty}(\Omega)\right), \\
& w \in \mathscr{C}\left(\left(0, T_{U_{0}}\right], \mathcal{D}(\Lambda)\right) \cap \mathscr{C}\left(\left[0, T_{U_{0}}\right], L_{+}^{2}(\Omega)\right) \cap \mathscr{C}^{1}\left(\left(0, T_{U_{0}}\right], L_{+}^{2}(\Omega)\right) .
\end{aligned}
$$

3.3 Global solutions generating a continuous dynamical system

After establishing the existence and uniqueness of local solutions to the reactiondiffusion system (25), we can now prove that these solutions enjoy a dissipative estimate. Indeed, there exist two positive coefficients $k_{1}, k_{2}$ such that

$$
\left\|U\left(t, U_{0}\right)\right\|_{X} \leq k_{1}\left[e^{-k_{2} t}\left\|U_{0}\right\|_{X}+1\right], \quad t \leq T_{U_{0}},
$$

for any $U_{0} \in X_{0}$, where $U\left(t, U_{0}\right)$ denotes the solution of system (25) starting from $U_{0}$, defined on $\left[0, T_{U_{0}}\right]$. Since the proof of estimate (28) is quite similar to that of Proposition 11.1 in [40], we may omit it. Estimate (28) guarantees that the local solutions are global in time, and proves that the reduced system (25) generates a continuous dynamical system $\mathscr{S}(t)$ defined in $X$, with phase space $X_{0}$. More precisely, $\mathscr{S}(t)$ is defined by:

$$
\mathscr{S}(t) U_{0}=U\left(t, U_{0}\right),
$$

for any $U_{0} \in X_{0}$ and all $t \geq 0$. In the sequel, we aim to analyze the equilibrium states of the continuous dynamical system $\mathscr{S}(t)$.

\section{Stability analysis of the reaction-diffusion-advection system}

4.1 Equilibrium states of the water-forest system

Geographical and hydrological observations of several forest areas, such as the Amazon basin or the Congo basin, suggest that the water-forest ecosystem often reaches a stationary equilibrium. It is the purpose of this section to 
investigate the existence of such stationary solutions to system (5). More precisely, we aim to firstly characterize stationary homogeneous solutions, which are constant in time and uniform in space, and secondly investigate the existence of stationary heterogeneous solutions, which are constant in time but not necessarily uniform in space. Next, we explore the stability of a particular stationary heterogeneous solution by applying spectral theory methods.

\subsubsection{Stationary homogeneous solutions}

We begin with the research of stationary homogeneous solutions of system (5), which are constant in time and uniform in space. Propositions 1 and 2 below establish the list of these particular solutions.

Let us denote by $V^{*}=\left(\rho^{*}, u^{*}, v^{*}, w^{*}\right)^{T}$ a stationary homogeneous solution of system (5). Such a stationary homogeneous solution satisfies the following system:

$$
\left\{\begin{aligned}
\rho^{*} & =m(x), \\
\sigma \rho^{*} & =\varphi\left(\rho^{*}\right) v^{*}, \\
\beta \delta w^{*} & =\left[\gamma\left(v^{*}, \rho^{*}\right)+f\right] u^{*}, \\
f u^{*} & =h\left(\rho^{*}\right) v^{*}, \\
\beta w^{*} & =\alpha\left(\rho^{*}\right) v^{*} .
\end{aligned}\right.
$$

The first equation of the latter system imposes a condition on the boundary term $m(x)$. Thus system (5) cannot admit any stationary homogeneous solution, unless the boundary term $m$ is itself uniform on the inflow boundary $\Gamma$.

First case: vanishing oceanic contribution. Let us assume that $m(x)=0$ for all $x \in \Gamma$. This situation models a vanishing oceanic contribution. Note that this situation happens with a seasonal cyclic rhythm in arctic regions, thus can be of great biological interest. Then system (30) becomes

$$
\left\{\begin{aligned}
\rho^{*} & =0 \\
0 & =\varphi(0) v^{*}, \\
\beta \delta w^{*} & =\left[\gamma\left(v^{*}, 0\right)+f\right] u^{*}, \\
f u^{*} & =h(0) v^{*}, \\
\beta w^{*} & =\alpha(0) v^{*} .
\end{aligned}\right.
$$

Since $\alpha(0)=0$, we obtain $w^{*}=0$, which leads to $u^{*}=v^{*}=0$. In other words, the trivial solution $(0,0,0,0)$ is a stationary homogeneous solution to system (5) under the assumption $m \equiv 0$. We obtain the following proposition.

Proposition 1 If $m(x)=0$ for all $x \in \Gamma$, then system (5) admits a unique stationary homogeneous solution $V^{*}=(0,0,0,0)$. 
Second case: uniform oceanic contribution. Let us now assume that $m(x)=m^{*}>0$ for all $x \in \Gamma$. With this assumption, system (30) becomes

$$
\left\{\begin{aligned}
\rho^{*} & =m^{*} \\
\sigma m^{*} & =\varphi\left(m^{*}\right) v^{*}, \\
\beta \delta w^{*} & =\left[\gamma\left(v^{*}, m^{*}\right)+f\right] u^{*}, \\
f u^{*} & =h\left(m^{*}\right) v^{*} \\
\beta w^{*} & =\alpha\left(m^{*}\right) v^{*} .
\end{aligned}\right.
$$

Assume for simplicity that $\alpha, \varphi, \mu$ and $h$ are given by expressions (42) below; then we obtain successively

$$
\begin{aligned}
& v^{*}=\frac{\sigma m^{*}}{\varphi\left(m^{*}\right)}=\frac{\sigma}{\varphi_{0}}\left(1+m^{*}\right), \\
& u^{*}=\frac{h\left(m^{*}\right) v^{*}}{f}=\frac{\sigma}{f \varphi_{0}}\left(h_{0}+h_{1} m^{*}\right), \\
& w^{*}=\frac{\alpha\left(m^{*}\right) v^{*}}{\beta}=\frac{\alpha_{0} \sigma}{\beta \varphi_{0}} m^{*},
\end{aligned}
$$

and $m^{*}$ must satisfy

$$
f \delta \alpha_{0} m^{*}=\left[a\left(\frac{\sigma}{\varphi_{0}}\left(1+m^{*}\right)-b\right)^{2}+c+f+\frac{\mu_{0}}{1+m^{*}}\right]\left(h_{0}+h_{1} m^{*}\right),
$$

or equivalently

$$
P\left(m^{*}\right)=0
$$

where $P(m)$ is given by:

$$
\begin{aligned}
P(m) & =\left\{\left[a\left(\frac{\sigma}{\varphi_{0}}(1+m)-b\right)^{2}+c+f\right](1+m)+\mu_{0}\right\}\left(h_{0}+h_{1} m\right) \\
& -f \delta \alpha_{0} m(1+m) .
\end{aligned}
$$

Each positive solution of the latter fourth degree polynomial equation leads to a stationary homogeneous solution to system (5). Since $P(0)>0$ and $\lim _{m \rightarrow+\infty} P(m)=+\infty, P(m)$ admits 0,2 or 4 positive roots, depending on the values of the parameters involved in the model (5). However, we observe that

$$
\begin{aligned}
& P(-1)=\mu_{0}\left(h_{0}-h_{1}\right)<\mu_{0} h_{0} \\
& P(0)=\left[a\left(\frac{\sigma}{\varphi_{0}}-b\right)^{2}+c+f+\mu_{0}\right] h_{0}>\mu_{0} h_{0},
\end{aligned}
$$

from which we can deduce $P(-1)<P(0)$. Now, if $P(m)$ admits 4 positive roots, then it necessarily implies $P(-1)>P(0)$, which leads to a contradiction. Therefore, the number of positive roots of $P(m)$ is 0 or 2 (both cases are depicted in figure 4). 


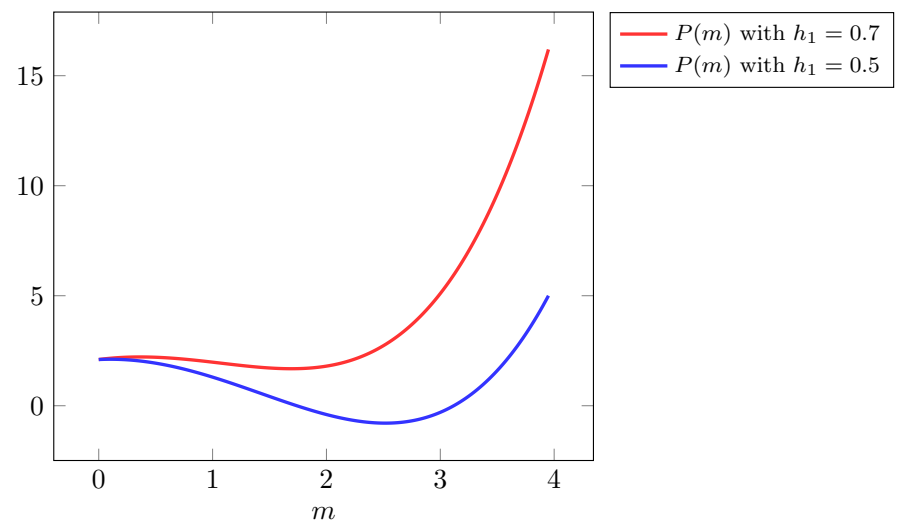

Fig. 4 Shape of the curve $P(m)$, where $P$ is given by (34), for the parameters values $a=c=0.1, \sigma=\varphi_{0}=b=f=\mu_{0}=h_{0}=\alpha_{0}=1$ and $\delta=1.9$. If $h_{1}=0.5$, then $P(m)$ admits 2 positive roots, which corresponds to 2 stationary homogeneous solutions of the form (35). If $h_{1}=0.7$, then $P(m)$ has no positive root, thus system (5) has no stationary solution of the form (35).

Proposition 2 Assume that $\alpha, \varphi, \mu$ and $h$ are given by expressions (42). Then each positive solution $m^{*}$ of the fourth degree polynomial equation (33) generates a stationary homogeneous solution $\left(\rho^{*}, u^{*}, v^{*}, w^{*}\right)$ to system (5), which can be parametrized as follows:

$$
\begin{aligned}
\rho^{*} & =m^{*}, \\
v^{*} & =\frac{\sigma}{\varphi_{0}}\left(1+m^{*}\right), \\
u^{*} & =\frac{\sigma}{f \varphi_{0}}\left(h_{0}+h_{1} m^{*}\right), \\
w^{*} & =\frac{\alpha_{0} \sigma}{\beta \varphi_{0}} m^{*} .
\end{aligned}
$$

Remark 2 The stationary homogeneous solutions of system (5), whose existence has been proved in Propositions 1 and 2, correspond to equilibrium states of the water-forest ecosystem. In particular, we have shown that our model is able to reproduce the situation of a uniform spatial distribution of the water resource, which is verified for several rain forests of continental scale. Furthermore, considering that the oceanic contribution to the water cycle is a given parameter, we can view the polynomial equation (33) as an equation whose unknowns are the parameters of the forest model (5). For example, equation (34) is a simple first degree polynomial equation of unknown $f$ (aging rate of young trees), which suggests that the forest ecosystem may be regulating its own biological characteristics in order to reach a healthy equilibrium. 


\subsubsection{Stationary heterogeneous solutions}

We continue with the research of stationary heterogeneous solutions of system (5), which are constant in time but not necessarily uniform in space. These stationary heterogeneous solutions can be written

$$
\bar{V}(x)=(\rho(x), u(x), v(x), w(x)) ;
$$

they satisfy the following system:

$$
\left\{\begin{array}{rlrl}
\boldsymbol{a} \cdot \nabla \rho+\sigma \rho & =\varphi(\rho) v, & x & \in \Omega, \\
\beta \delta w & =\gamma(v, \rho) u+f u, & x \in \Omega, \\
f u & =h(\rho) v, & x & \in \Omega, \\
\beta w & =d \Delta w+\alpha(\rho) v, & x & \in \Omega, \\
\frac{\partial w}{\partial \nu}(x) & =0, & & x \in \partial \Omega, \\
\rho(x) & =m(x), & & x \in \Gamma .
\end{array}\right.
$$

Solutions of particular interest are such that $u, v, w$ are stationary homogeneous, while $\rho=\rho(x)$ is stationary heterogeneous, or vice versa. At least one such solution exists, when

$$
u=v=w \equiv 0
$$

In that case, $\rho=\rho(x)$ satisfies an advection equation, as stated in the following proposition.

Proposition 3 System (5) admits a particular stationary heterogeneous solution denoted by $(\bar{\rho}(x), 0,0,0)$, where $\bar{\rho}(x)$ satisfies the stationary advection equation

$$
\boldsymbol{a} \cdot \nabla \bar{\rho}+\sigma \bar{\rho}=0, x \in \Omega, \quad \bar{\rho}(x)=m(x), x \in \Gamma .
$$

By virtue of Theorem $1, \bar{\rho}(x)$ is determined along the characteristic lines of the advection field $\boldsymbol{a}$ by:

$$
\bar{\rho}(x)=m\left(x_{0}\right) e^{-\sigma s}, \quad x \in \Omega,
$$

where $x_{0}=\zeta_{1}(x)$ and $s=\zeta_{2}(x)$ (see equation (17)).

Remark 3 The stationary heterogeneous solution $(\bar{\rho}(x), 0,0,0)$ presented in the latter proposition models the situation of a deforested area. Hence, it is of great interest to study the stability of that solution. In the present situation, stability would correspond to the impossibility to recreate a healthy forest ecosystem with small perturbations of the stationary heterogeneous solution $(\bar{\rho}(x), 0,0,0)$, which could for example correspond to reforestation. At the opposite, instability would signify that a small change in the land-use of the deforested area could lead to the regenerateness of the forest.

Remark 4 It is worth noting that other stationary heterogeneous solutions may exist. In particular, some of these stationary heterogeneous solutions can be discontinuous. Such discontinuous solutions have been constructed in [22], but for the model without water resource. 
4.2 Stability analysis

In this section, we investigate the stability of the stationary heterogeneous solution $(\bar{\rho}(x), 0,0,0)$ given by Proposition 3 . We denote by $\bar{U}=(0,0,0)$ the corresponding stationary solution of the reduced system (25), with the property $\bar{\rho}(x)=\psi(0)$. Our stability analysis is based on spectral methods; it differs from that presented in [22] since the operator $\bar{A}=A-F^{\prime}(\bar{U})$, where $F^{\prime}(U)$ denotes the derivative of the non-linear operator $F$, will admit non constant coefficients. In order to avoid any confusion with the parameters $\sigma$ and $\rho$ of the water-forest model (5), the spectrum of any operator $B$ will be denoted by $\operatorname{Spec}(B)$ and its resolvent set will be denoted by $R(B)$. Here, the derivative $F^{\prime}(U)$ is given by

$$
F^{\prime}(U)=\left[\begin{array}{ccc}
-\gamma(v, \psi(v))-2 a u(v-b)-u \mu^{\prime}(\psi(v)) D \psi(v) & \beta \delta \\
f & 1-v h^{\prime}(\psi(v)) D \psi(v)-h(\psi(v)) & 0 \\
0 & v \alpha^{\prime}(\psi(v)) D \psi(v)+\alpha(\psi(v)) & 0
\end{array}\right] .
$$

Since $\bar{\rho}(x)=\psi(0)$, we obtain after elementary computations:

$$
\bar{A}=\left[\begin{array}{ccc}
f+a b^{2}+c+\mu(\bar{\rho}(x)) & 0 & -\beta \delta \\
-f & h(\bar{\rho}(x)) & 0 \\
0 & -\alpha(\bar{\rho}(x)) & -d \Delta+\beta
\end{array}\right] .
$$

Next, the stability of $\bar{U}$ is determined by the location of the spectrum $\operatorname{Spec}(\bar{A})$ of $\bar{A}$ with respect to the line $i \mathbb{R}=\{z \in \mathbb{C}: \Re(z)=0\}$. We introduce the quantities $\omega_{1}>0$ and $\omega_{2}>0$ given by

$$
\omega_{1}=f+a b^{2}+c+\min _{x \in \bar{\Omega}} \mu(\bar{\rho}(x)), \quad \omega_{2}=\min _{x \in \bar{\Omega}} h(\bar{\rho}(x)),
$$

as well as $\omega_{0}=\frac{1}{2} \min \left(\omega_{1}, \omega_{2}\right)>0$. Let us now prove the following lemma.

Lemma 1 Consider $\Sigma=\operatorname{Spec}(-\bar{A}) \cap\left\{z \in \mathbb{C}: \Re(z) \geq-\omega_{0}\right\}$. Then the set $\Sigma$ enjoys the following properties.

(i) One has

$$
\Sigma=\left\{\lambda \in \mathbb{C}: \Re(\lambda) \geq-\omega_{0} \text { and } 0 \in \operatorname{Spec}\left(L_{\lambda}\right)\right\},
$$

wherein the elliptic operator $L_{\lambda}: \mathcal{D}\left(L_{\lambda}\right) \subset L^{2}(\Omega) \rightarrow L^{2}(\Omega)$ is given, for all complex numbers $\lambda$ with $\Re(\lambda) \geq \omega_{0}$, by

$$
\mathcal{D}\left(L_{\lambda}\right)=H_{N}^{2}(\Omega) \text { and } L_{\lambda}=\lambda+\beta-d \Delta-M_{\lambda}(x)
$$

and where the function $M_{\lambda} \in C^{0}(\bar{\Omega})$ is defined by

$$
M_{\lambda}(x)=\frac{f \beta \delta \alpha(\bar{\rho}(x))}{[\lambda+h(\bar{\rho}(x))]\left[\lambda+f+a b^{2}+c+\mu(\bar{\rho}(x))\right]} .
$$

(ii) One has $\Sigma \subset \mathbb{R}$. 
Proof Let us first observe that when $\lambda \in \mathbb{C}$ with $\Re(\lambda) \geq-\omega_{0}$, then both functions

$$
x \mapsto \frac{1}{\lambda+h(\bar{\rho}(x))} \text { and } x \mapsto \frac{1}{\lambda+f+a b^{2}+c+\mu(\bar{\rho}(x))},
$$

are continuous on $\bar{\Omega}$.

In order to prove $(i)$, namely to describe the spectrum of $-\bar{A}$ in the half plane $\left\{z \in \mathbb{C}: \Re(z) \geq-\omega_{0}\right\}$, let us fix a complex number $\lambda$ in this half plane and solve the equation

$$
(\lambda+\bar{A}) U=Y,
$$

for $Y=\left(f_{1}, f_{2}, f_{3}\right) \in X$ and $U=(u, v, w) \in L^{\infty}(\Omega) \times L^{\infty}(\Omega) \times H_{N}^{2}(\Omega)$. This rewrites

$$
\left\{\begin{array}{l}
{\left[\lambda+f+a b^{2}+c+\mu(\bar{\rho}(x))\right] u-\beta \delta w=f_{1}} \\
-f u+[\lambda+h(\bar{\rho}(x))] v=f_{2} \\
\alpha(\bar{\rho}(x)) v+(\lambda+\beta-d \Delta) w=f_{3}
\end{array}\right.
$$

or equivalently

$$
\left\{\begin{array}{l}
L_{\lambda} w=c_{1}(x) f_{1}+c_{2}(x) f_{2}+c_{3}(x) f_{3} \\
u=\left(f_{1}+\beta \delta w\right) /\left[\lambda+f+a b^{2}+c+\mu(\bar{\rho}(x))\right] \\
v=\left(f_{2}+f u\right) /[(\lambda+h(\bar{\rho}(x))]
\end{array}\right.
$$

wherein the functions $c_{1}, c_{2}$ and $c_{3}$ are given by

$$
\begin{aligned}
& c_{1}(x)=\alpha(\bar{\rho}(x)), \\
& c_{2}(x)=\alpha(\bar{\rho}(x))\left[\lambda+f+a b^{2}+c+\mu(\bar{\rho}(x))\right], \\
& c_{3}(x)=\left[\lambda+f+a b^{2}+c+\mu(\bar{\rho}(x))\right][\lambda+h(\bar{\rho}(x))] .
\end{aligned}
$$

As a consequence of the above computations, one obtains for each $\lambda \in\{z \in$ $\left.\mathbb{C}: \Re(z) \geq-\omega_{0}\right\}, \lambda \in R(-\bar{A})$ if and only if $0 \in R\left(L_{\lambda}\right)$, which completes the proof of $(i)$.

Let us now prove $(i i)$, that is, $\Sigma \subset \mathbb{R}$. To see this, let $\lambda \in \Sigma$ be given. Then since $0 \in \operatorname{Spec}\left(L_{\lambda}\right)$, there exists $w \in H_{N}^{2}(\Omega) \backslash\{0\}$ such that $L_{\lambda} w=0$.

Multiplying this identity by the conjugate function $\bar{w} \in H_{N}^{2}(\Omega)$ and integrating over $\Omega$ yields

$$
d \int_{\Omega}|\nabla w(x)|^{2} d x+(\lambda+\beta) \int_{\Omega}|w(x)|^{2} d x-\int_{\Omega} M_{\lambda}(x)|w(x)|^{2} d x=0 .
$$

Next the imaginary part of the above equation reads as

$$
\Im(\lambda) \int_{\Omega}|w(x)|^{2} d x-\int_{\Omega} \Im\left(M_{\lambda}(x)\right)|w(x)|^{2} d x=0 .
$$

Now set for simplicity

$$
a(x)=f+a b^{2}+c+\mu(\bar{\rho}(x)),
$$


so that

$$
\Im\left(M_{\lambda}(x)\right)=-\Im(\lambda) \frac{f \beta \delta \alpha(\bar{\rho}(x))}{|\lambda+h(\bar{\rho}(x))|^{2}|\lambda+a(x)|^{2}}[2 \Re(\lambda)+h(\bar{\rho}(x))+a(x)],
$$

and (39) rewrites as

$$
\Im(\lambda) \int_{\Omega}|w(x)|^{2}\left[1+\frac{f \beta \delta \alpha(\bar{\rho}(x))[2 \Re(\lambda)+h(\bar{\rho}(x))+a(x)]}{|\lambda+h(\bar{\rho}(x))|^{2}|\lambda+a(x)|^{2}}\right] d x=0 .
$$

Finally, since $\Re(\lambda) \geq-\omega_{0}$, it follows that the integrand is positive and we end-up with $\Im(\lambda)=0$, which concludes the proof of the lemma.

For $\lambda \in\left[-\omega_{0}, \infty\right)$, define by $s(\lambda)$ the smallest eigenvalue of $L_{\lambda}$. Recalling that it is given by the Rayleigh quotient (see for instance [5], Theorem 2.1), we obtain

$$
s(\lambda)=\lambda+\beta+\min _{\varphi \in H^{1}(\Omega),\|\varphi\|_{L^{2}}=1}\left\{d \int_{\Omega}|\nabla \varphi|^{2} d x-\int_{\Omega} M_{\lambda}(x) \varphi^{2}(x) d x\right\} .
$$

Let us observe that $s:\left[\omega_{0}, \infty\right) \rightarrow \mathbb{R}$ is continuous, non-decreasing and satisfies $s(\lambda) \sim \lambda$ as $\lambda \rightarrow \infty$.

We obtain the following theorem.

Theorem 3 If $s(0)>0$, then there exists $\varepsilon>0$ small enough such that

$$
\operatorname{Spec}(-\bar{A}) \subset\{z \in \mathbb{C}: \Re(z) \leq-\varepsilon\},
$$

and the stationary state $(\bar{\rho}(x), 0,0,0)$ is locally stable.

If $s(0)<0$, then there exists $\lambda_{0}>0$ such that $\lambda_{0} \in \operatorname{Spec}(-\bar{A})$ and the stationary state $(\bar{\rho}(x), 0,0,0)$ is unstable.

Proof The proof Theorem 3 is given in the appendix.

The stability condition for the equilibrium $(\bar{\rho}(x), 0,0,0)$ is therefore related to the sign of the quantity $s(0)$ that reads as

$$
s(0)=\beta+\min _{\varphi \in H^{1}(\Omega),\|\varphi\|_{L^{2}}=1}\left\{d \int_{\Omega}|\nabla \varphi|^{2} d x-\int_{\Omega} M_{0}(x) \varphi^{2}(x) d x\right\},
$$

where $M_{0}(x)$ is expressed, thanks to (38), by

$$
M_{0}(x)=\frac{f \beta \delta \alpha(\bar{\rho}(x))}{h(\bar{\rho}(x))\left[f+a b^{2}+c+\mu(\bar{\rho}(x))\right]} .
$$

Now the explicit expression of $\bar{\rho}(x)$ given by (37) and equations (40)(41) allow to discuss the stability of the stationary heterogeneous solution $(\bar{\rho}(x), 0,0,0)$ in terms of the size of the domain $\Omega$, the exponential decrease rate $\sigma$ and of the oceanic contribution $m(x)$ to the water resource. Indeed, if 
the size of the domain $\Omega$ increases along the characteristic lines of the advection field $\boldsymbol{a}$, then the minimum of $\bar{\rho}(x)$ over $\Omega$ is attracted to zero; since $\alpha$ is an increasing function, whereas $\mu$ and $h$ are decreasing functions, we observe that $M_{0}(x)$ may decrease, and therefore $s(0)$ may remain positive. Similarly, if the rate $\sigma$ increases, or if the intensity of the oceanic contribution $m(x)$ decreases, then the same occurs. In these cases, the stationary heterogeneous solution $(\bar{\rho}(x), 0,0,0)$ is stable. At the contrary, if the size of $\Omega$ decreases along the characteristic lines of the advection field $\boldsymbol{a}$, if the rate $\sigma$ decreases, or if the intensity of the oceanic contribution $m(x)$ increases, then the minimum of $\bar{\rho}(x)$ over $\Omega$ grows, thus $s(0)$ is likely to become negative. If $s(0)$ decreases further, then the instability if $(\bar{\rho}(x), 0,0,0)$ is strengthened, since the number of positive values in $\operatorname{Spec}(-\bar{A})$ increases.

Roughly speaking, a large domain or a weak intensity of the water resource are seen to stabilize the stationary heterogeneous solution $(\bar{\rho}(x), 0,0,0)$, which corresponds to a deforested area; in that case, the regenerateness of the forest ecosystem seems to be compromised. It is the purpose of the next section to underpin and illustrate by numerical simulations this theoretical stability analysis.

\section{Numerical simulations}

In this section, we propose to illustrate, by a selection of relevant numerical simulations, several qualitative properties of the water-forest model given by system (5), which allows to test an infinite number of scenarios. First, we aim to show that our model fits well with the exponential decrease principle of water precipitation for deforested areas. Then, we investigate the stability of the stationary heterogeneous solution given in Proposition 3, which models such a deforested area. We exhibit various parameter regimes for which the latter solution is stable, which means that any small perturbation of the deforested equilibrium fails to recover the forest ecosystem, and other parameter regimes for which it is unstable, which corresponds to the possible regenerateness of the forest ecosystem after small changes. We also investigate the effect of a small climate change on this regenerateness process and prove that a small variation of the oceanic contribution to the water cycle can compromise the regenerateness of the forest ecosystem. Finally, we show that our model allows to test multiple scenarios of deforestation, by analyzing the effect of three deforestation strategies on the equilibrium of the water-forest ecosystem.

All the computations have been performed in a Debian/Gnu-Linux environment with the FreeFem++ software [15]. The calculation code is based on a splitting numerical scheme of Strang type [35] with discretization of time by finite differences and discretization of space by finite elements, in which the advection-diffusion process and the reaction process are separated. The values of the main parameters are normalized and listed in table 2 . We assume that the domain $\Omega$ is given by an ellipse of dimensions $2 L$ and $L$, with $L=2$, as illustrated in figure 1. The advection field $\boldsymbol{a}$ is simply directed along the 
first component axis and given by $a_{1}(x)=-0.5, a_{2}(x)=0$, which models the predominance of single directed winds, as observed in several tropical regions. However, other atmospheric fields could easily be considered. The oceanic border $\Gamma$ corresponds in this case to the right-side of the domain $\Omega$. Finally, the following explicit expressions for the functions $\alpha, \varphi, \mu$ and $h$ have been considered (see figure 3):

$$
\alpha(\rho)=\frac{\alpha_{0} \rho}{1+\rho}, \quad \varphi(\rho)=\frac{\varphi_{0} \rho}{1+\rho}, \quad \mu(\rho)=\frac{\mu_{0}}{1+\rho}, \quad h(\rho)=\frac{h_{0}+h_{1} \rho}{1+\rho} .
$$

Table 2 Values of the main parameters of the forest model (5) for the numerical simulations.

\begin{tabular}{ll}
\hline Parameter & Value \\
\hline$\alpha_{0}$ & 0.9 \\
$\mu_{0}$ & 0.6 \\
$\beta$ & 0.6 \\
$\delta$ & 0.9 \\
$f$ & 1.0 \\
$h_{0}$ & 0.15 or 0.5 \\
$h_{1}$ & 0.05 \\
$\sigma$ & 0.7 \\
$\varphi_{0}$ & 0.8 \\
$a$ & 0.01 \\
$b$ & 1 \\
$c$ & 0.1 \\
$d$ & 10 \\
\hline
\end{tabular}

5.1 Exponential decrease of the water resource in deforested areas

As mentioned previously, the stationary heterogeneous solution $(\bar{\rho}(x), 0,0,0)$ given in Proposition 3 reproduces the real-world situation of a deforested area, in which it is observed that precipitation decreases at an exponential rate with the distance to ocean. However, this exponential decrease principle can hide a variety of spatial distributions of the water resource, depending on the oceanic contribution, which corresponds to the boundary term $m(x)$ in our model, as well as the atmospheric activity, which is determined by the advection field $\boldsymbol{a}$ in system (5).

The three numerical simulations presented in figure 5 are obtained with $m(x)=1-x_{2}^{2}, m(x)=1$ and $m(x)=1+x_{2}$ respectively, for all $x \in \Gamma$. The first situation models an atmospheric activity which is maximal in the center of the littoral; the second situation corresponds to a uniform oceanic contribution (such uniform oceanic contributions have been proved to lead to stationary homogeneous solutions in Proposition 2); finally, the third situation models an 
(a) $\rho(x)$ with $m(x)=1-x_{2}^{2}$

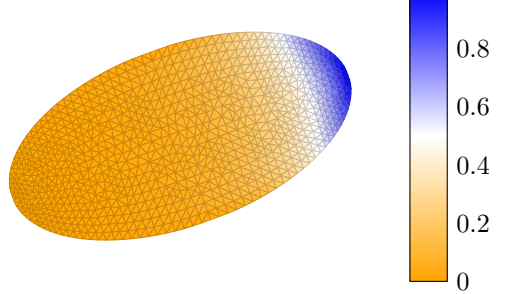

(b) $\rho(x)$ with $m(x)=1$

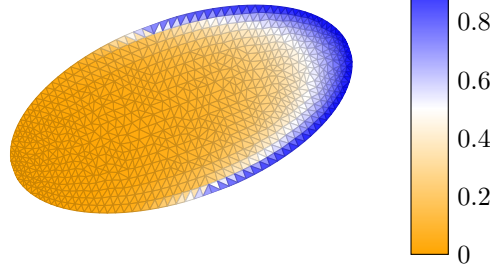

(c) $\rho(x)$ with $m(x)=1+x_{2}$

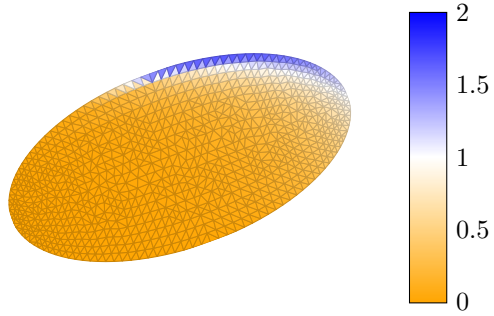

Fig. 5 Spatial distribution of the water resource $\rho(x)$ for the stationary heterogeneous solution $(\rho(x), 0,0,0)$, which models a deforested area. The distribution has been calculated for three distinct oceanic distributions : (a) $m(x)=1-x_{2}^{2}$; (b) $m(x)=1$; (c) $m(x)=1+x_{2}$. In each case, the exponential decrease of the water resource is verified.

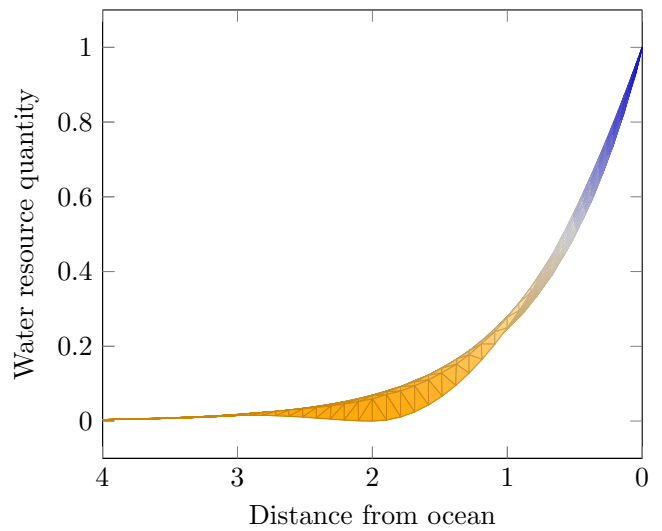

Fig. 6 Exponential decrease of the water resource with respect to the distance from the oceanic littoral.

atmospheric activity which is maximal at the top of the littoral. In each case, the exponential decrease of the water resource is obviously verified. A different view of the spatial distribution of the water resource is presented in figure 6 , so as to visualize this exponential decrease. We emphasize that transitions 
from one case to another can be provoked by climate perturbations which change the atmospheric activity. In particular, these climate perturbations can make stationary homogeneous solutions vanish, and thus compromise the equilibrium states of the water forest ecosystem, as will be shown below.

5.2 Stability of the heterogeneous stationary solution: death of the forest ecosystem

Here, we propose to exhibit a non trivial parameter regime for which the stationary homogeneous solution $(\bar{\rho}(x), 0,0,0)$ given in Proposition 3 is locally stable. Such a local stability means that a small perturbation of the deforested area will generate a new solution of the water-forest model (5), which will again be attracted to the deforested equilibrium state. In other words, stability has to be understood as an impossibility to recreate favorable conditions for a regenerateness of the forest, by small actions such as reforestation.

We present in figure 7 a numerical simulation of our model, obtained with an heterogeneous oceanic contribution given by $m(x)=1-0.7 x_{2}^{2}$ for all $x \in \Gamma$. The initial distribution of young trees $u_{0}$ is defined by

$$
u_{0}\left(x_{1}, x_{2}\right)=\frac{1}{1+0.1\left(x_{1}-\frac{3 L}{4}\right)^{2}+0.1 x_{2}^{2}},
$$

whereas the initial distributions of old trees $v_{0}$ and of seeds $w_{0}$ are given by $v_{0} \equiv 0$ and $w_{0} \equiv 0$. This scenario roughly models reforestation near the littoral. The values of other parameters are given in table 2 . The maximum mortality rate of old trees is set to $h_{0}=0.5$. In this numerical simulation, we observe that the density of young trees decreases with time: the maximum density is of order 1 at time $t=0$, or order $10^{-1}$ at time $t=20$, and of order $10^{-2}$ at time $t=100$. After 100 units of time, the distribution of young trees is limited to a narrow area near the littoral. In background, the spatial distribution of old trees follows the same evolution, thus is unable to aliment the water cycle through forest transpiration, and unable to produce a sufficient quantity of seeds so as to maintain a sufficient birth of young trees. Therefore, the mortality rate of old trees is too high to regenerate the forest ecosystem.

5.3 Instability of the stationary heterogeneous solution: regenerateness of the forest

We continue our stability analysis of the stationary heterogeneous solution $(\bar{\rho}(x), 0,0,0)$ given in Proposition 3, with the research of non trivial parameter regimes which guaranty instability. In this case, instability is seen as the opportunity to recreate a healthy forest ecosystem by small perturbations of the deforested distribution $(\bar{\rho}(x), 0,0,0)$; these small perturbations can model reforestation actions. 
$\rho(x)$ at $t=0$

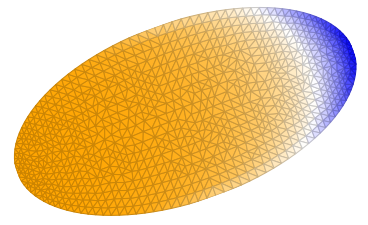

$\rho(x)$ at $t=20$

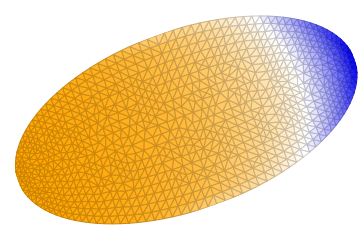

$\rho(x)$ at $t=100$

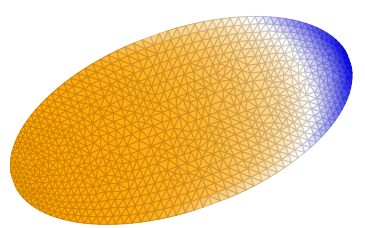

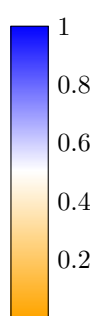
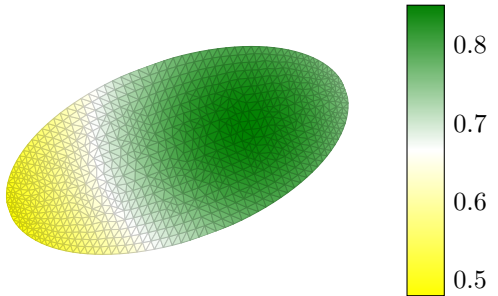

$u(x)$ at $t=20$

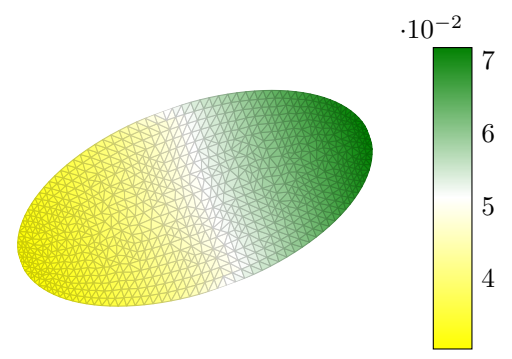

$u(x)$ at $t=100$

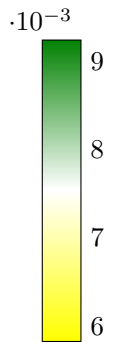

Fig. 7 Time evolution of the spatial distribution of the water resource $\rho(x)$ and of the young trees density $u(x)$, for a parameter regime which leads to the local stability of the deforested equilibrium distribution. This local stability means that a small perturbation of the deforested area generates a new solution of the water-forest model (5), which is again be attracted to the deforested equilibrium state.

We present in figure 8 another numerical simulation of our model (5); the oceanic contribution $m(x)$ is similar to the previous section, as well as the initial distributions $u_{0}, v_{0}$ and $w_{0}$. The only change is in the parameter $h_{0}$ which models the maximum death rate of old trees: here, we set $h_{0}=0.15$. With this parameter value, we observe that the density of young trees $u(x)$ increases, after a transitional phase during which $u(x)$ is concentrated near the littoral. After about 580 units of time, the density of young trees $u(x)$ persists in the whole domain $\Omega$. In parallel, the density of old trees $v(x)$ follows the 
$\rho(x)$ at $t=0$

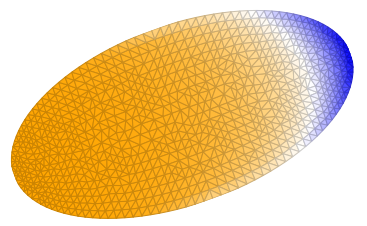

$\rho(x)$ at $t=520$

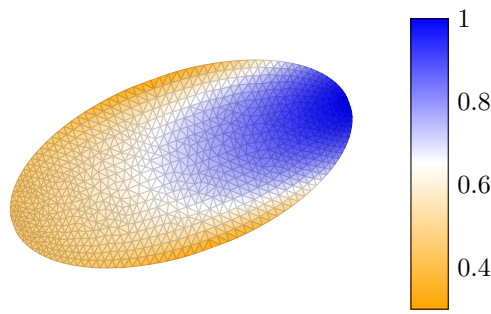

$\rho(x)$ at $t=580$

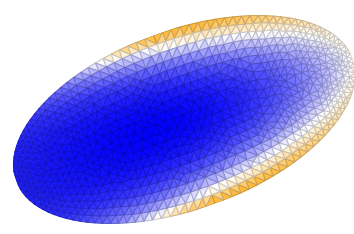

$u(x)$ at $t=0$
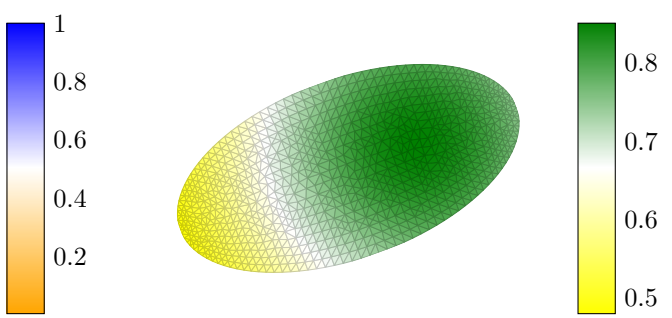

$u(x)$ at $t=520$

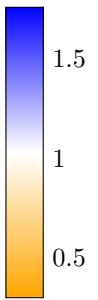

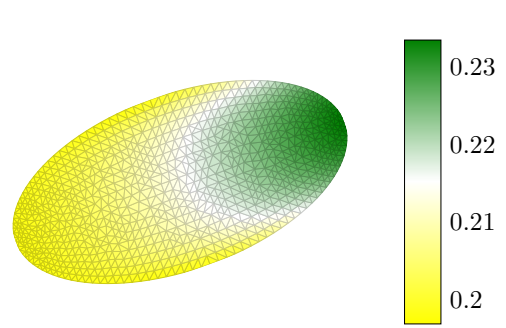

$u(x)$ at $t=580$

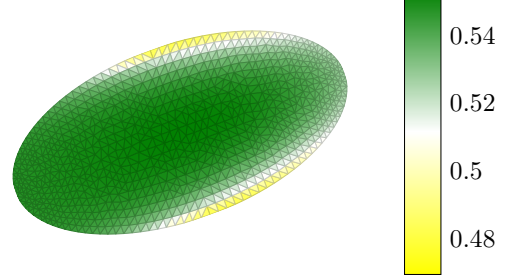

Fig. 8 Time evolution of the spatial distribution of the water resource $\rho(x)$ and of the young trees density $u(x)$, for a parameter regime which leads to the local instability of the deforested equilibrium distribution. Instability means that a small perturbation of the deforested area generates a new solution of the water-forest model (5), which converges to a healthy equilibrium state of the forest ecosystem.

same evolution. Its lower mortality rate $h_{0}$ now guarantees a sufficient forest transpiration, which accounts for the time evolution of the water resource $\rho(x)$ : after 580 units of time, $\rho(x)$ persists in the whole domain, and even admits greater values at the opposite of the littoral border.

These numerical simulations show that system (5) undergoes a bifurcation of loss of stability, with respect to a variation of the parameter $h_{0}$ : when $h_{0}$ overcomes a critical value $h_{0}^{c}$, then the stationary heterogeneous solution $(\bar{\rho}(x), 0,0,0)$ is seen to be locally stable; when $h_{0}$ is lesser than $h_{0}^{c}$, we observe that it looses its stability. Further numerical investigations show that 
Table 3 Effect of a variation of several parameters on the stability of the stationary heterogeneous solution $(\bar{\rho}(x), 0,0,0)$.

\begin{tabular}{lll}
\hline Parameter & Variation & Effect \\
\hline$h_{0}$ & decrease & loss of stability \\
$\delta$ & increase & loss of stability \\
$f$ & increase & loss of stability \\
$\alpha_{0}$ & increase & loss of stability \\
\hline
\end{tabular}

the instability is more robust when $h_{0}$ tends to 0 . However, we do not know at this stage if other steady states of system (5) bifurcate from $(\bar{\rho}(x), 0,0,0)$ as it looses its stability.

Finally, we indicate that the stability of the stationary heterogeneous solution $(\bar{\rho}(x), 0,0,0)$ is similarly lost under the variation of other parameters of the model (see Table 3). For instance, the loss of stability is observed for an increase of the seed establishment parameter $\delta$, for an increase of the aging rate $f$, or for an increase of the maximum production rate of seeds $\alpha_{0}$.

\subsection{Cascade effects due to climatic perturbation}

As mentioned previously, the oceanic contribution to the water cycle, given by the border term $m(x)$ in system (5), can be perturbed by climate changes. Such climatic perturbations can in turn affect the dynamics of the waterforest ecosystem. In particular, climate perturbations can modify the parameter regimes of instability of the deforested equilibrium, and contrary a regenerateness process of the forest.

We present in figure 9 another numerical simulation of our model; the parameters values are the same as in the previous section, except for the oceanic contribution $m(x)$ : here, we set $m(x)=0.9-0.7 x_{2}^{2}$ instead of $m(x)=$ $1-0.7 x_{2}^{2}$, and $a_{1}(x)=-0.4$ instead of $a_{1}(x)=-0.5$. These variations can roughly model a decrease of the intensity of precipitation. We observe that the water-forest ecosystem, which should converge to a healthy equilibrium in absence of climate perturbation, is now attracted to a vanishing equilibrium of the forest: after 800 units of time, the maximal density of young trees is of order $10^{-3}$, whereas it would be of order 1 in absence of climate perturbation. In background, the density of old trees follows the same evolution, and thus cannot contribute to the forest transpiration process, which in turn favors the decrease of the water resource far from the littoral. Therefore, the dynamics of system (5) can account for complex cascade effects in climate changes.

\subsection{Testing scenarios of deforestation}

It is nowadays admitted that the equilibrium of forest ecosystems is threatened by anthropic activities such as mass deforestation, besides climate changes, 
$\rho(x)$ at $t=0$

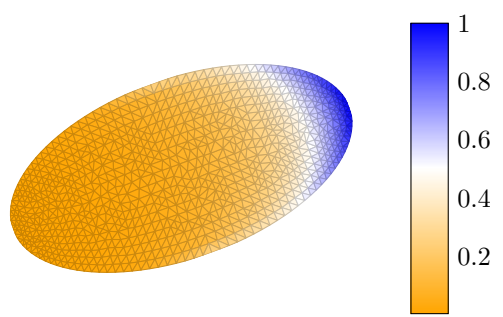

$\rho(x)$ at $t=80$

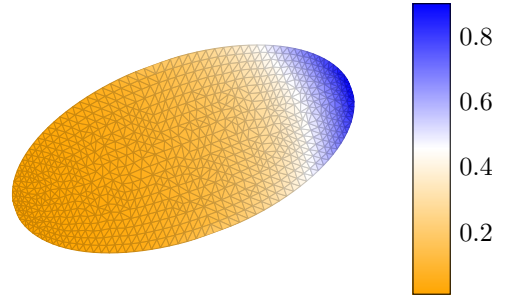

$\rho(x)$ at $t=800$

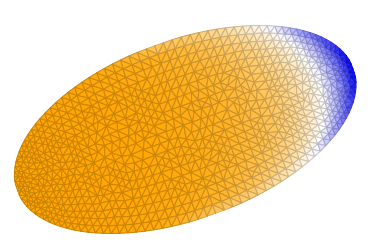

$u(x)$ at $t=0$

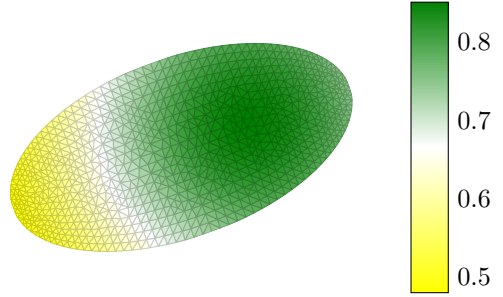

$u(x)$ at $t=80$

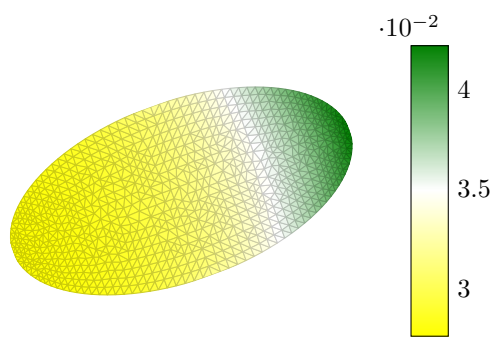

$u(x)$ at $t=800$

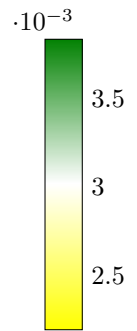

Fig. 9 Time evolution of the spatial distribution of the water resource $\rho(x)$ and of the young trees density $u(x)$, under a small perturbation of the oceanic contribution $m(x)$ of the water cycle, which affects the dynamics of the forest ecosystem. In absence of perturbation, the forest ecosystem would exhibit a regenerateness process, as depicted in figure 8 . Under the climatic perturbation, the system is attracted to the vanishing equilibrium of the ecosystem.

which can also have an anthropic origin. In this final section, we aim to show that our model allows to investigate the effect of deforestation on the water resource distribution, which in turn impacts the equilibrium of forest ecosystems. Here, we propose to test three scenarios of deforestation, which are schematized in figure 10 .

The first scenario corresponds to deforestation concentrated at the center of the forest area, far from the ocean. The second scenario models deforestation near the littoral. The third scenario tests the situation of several deforested patches. In each case, the deforested surfaces are of equivalent order. Obvi- 


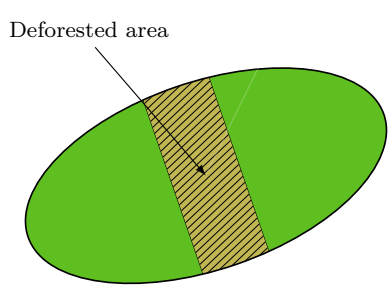

(a)

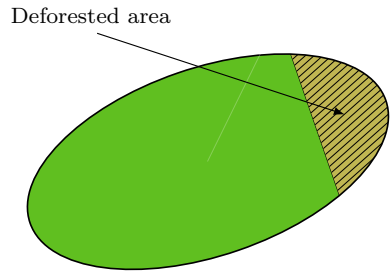

(b)

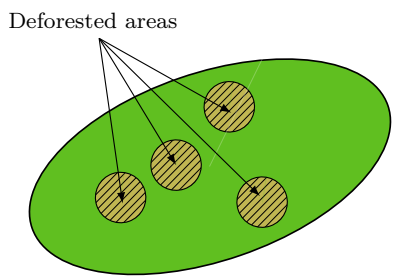

(c)

Fig. 10 Three scenarios of deforestation. (a) Deforestation at the center of the forest area. (b) Deforestation near the littoral. (c) Deforestation of several patches. In each case, the deforested surfaces are of equivalent order.

ously, deforestation may cause a perturbation in the forest evapotranspiration process, which is likely to affect the dynamics of the water cycle. For each scenario of deforestation, we can run the advection equation (2) in order to observe how the loss of forest evapotranspiration modifies the spatial distribution of water. The results are depicted in figure 11. We observe that the first and third scenarios seem to have severe impact on the distribution of the water resource $\rho(x)$, whereas the second scenario shows that the effect of deforestation seems to be damped. After running the advection equation (2), we can again run the whole model (5) in order to observe the time evolution of the water-forest ecosystem. We are then brought back to the above analysis of the dynamics of model (5): deforestation generates a new solution of the model; depending on the parameter regime, this new solution may be attracted to a vanishing equilibrium of the forest, as in section 5.2 , or at the opposite may converge to a healthy equilibrium of the forest, as in section 5.3. In the latter case, however, the dynamics of the forest ecosystem would be altered if the deforestation process persists and forbids a natural regenerateness of the forest. Once again, the cascade effects which exert a perturbation on the equilibrium of the ecosystem are reproduced by our mathematical model. 
(a)

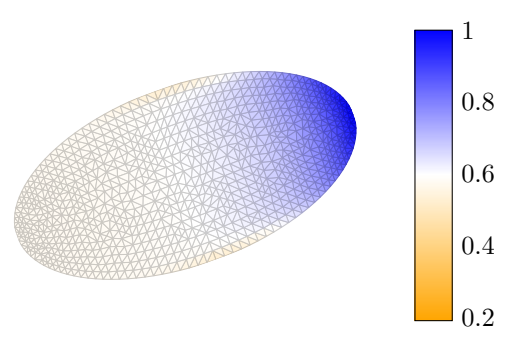

(c)

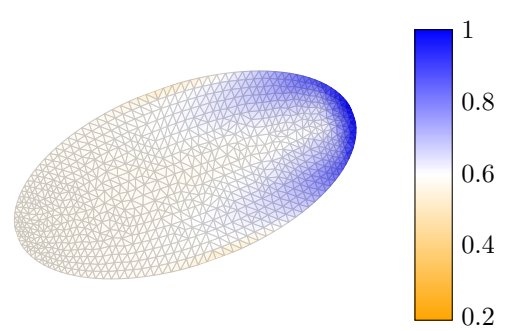

(b)

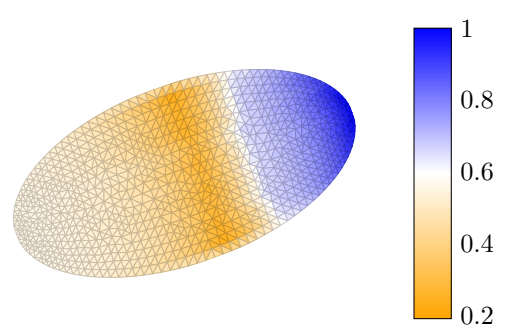

(d)

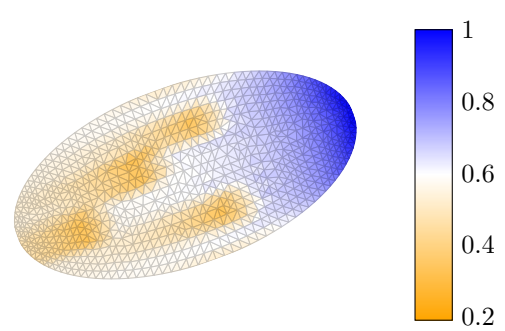

Fig. 11 Effect of deforestation on the spatial distribution of the water resource $\rho(x)$. (a) Distribution of $\rho(x)$ in absence of deforestation. (b) Spatial distribution of $\rho(x)$ in the first scenario (deforestation at the center of the area, figure 10(a)). (c) Spatial distribution of $\rho(x)$ in the second scenario (deforestation near the littoral, figure 10(b)). (d) Spatial distribution of $\rho(x)$ in the third scenario (deforestation of several patches, figure $10(\mathrm{c})$ ).

\section{Conclusion and perspectives}

In this work, we have presented an overview of an innovative mathematical model, which has been designed for studying the dynamics of forest ecosystems and the roles of the water resource and the atmospheric activity. Stemming from a well-known reaction-diffusion system, we have integrated the effect of the atmospheric activity by adding a moisture conservation equation, and the role of the water resource by considering biological parameters of the ecosystem as functions of the precipitation quantity. Our modeling approach results in a reaction-diffusion-advection model. This original model has been validated at two levels. Firstly, we have proved that it admits relevant stationary solutions; among these stationary solutions, the existence of homogeneous solutions shows that the model is able to reproduce the situation of almost uniform precipitation quantity over forested areas. Secondly, we have proved that our model is in concordance with the principle of exponential decrease of the water resource over deforested areas. Furthermore, the well-posedness of the model has been proved with a rigorous mathematical approach. Several numerical simulations have been presented so as to test the effects of climate change or deforestation on the equilibrium of the ecosystem. 
Finally, although abstract, our model can easily be adapted to real-world forests. Thus in a near future, we shall apply our model to the study case of the Amazon forest, after calibrating the parameters of system (5) with land use and precipitation statistical data.

\section{References}

1. W. R. Anderegg, A. T. Trugman, G. Badgley, A. G. Konings, and J. Shaw. Divergent forest sensitivity to repeated extreme droughts. Nature Climate Change, pages 1-5, 2020 .

2. M. Y. Antonovsky, R. Fleming, Y. A. Kuznetsov, and W. Clark. Forest-pest interaction dynamics: The simplest mathematical models. Theoretical Population Biology, 37(2):343-367, 1990.

3. D. B. Botkin et al. Forest dynamics: an ecological model. Oxford University Press on Demand, 1993.

4. G. Cantin and N. Verdière. Networks of forest ecosystems: Mathematical modeling of their biotic pump mechanism and resilience to certain patch deforestation. Ecological Complexity, 43:100850, 2020.

5. R. S. Cantrell and C. Cosner. Spatial ecology via reaction-diffusion equations. John Wiley \& Sons, 2004.

6. S. C. Cook-Patton, S. M. Leavitt, D. Gibbs, N. L. Harris, K. Lister, K. J. AndersonTeixeira, R. D. Briggs, R. L. Chazdon, T. W. Crowther, P. W. Ellis, et al. Mapping carbon accumulation potential from global natural forest regrowth. Nature, 585(7826):545$550,2020$.

7. C. Cosandey. Influence de la forêt sur le cycle de l'eau: conséquences d'une coupe forestière sur le bilan d'écoulement annuel. Hydrologie continentale, 7(1):13-22, 1992.

8. I. F. Creed, M. Weber, F. Accatino, and D. P. Kreutzweiser. Managing forests for water in the anthropocene - the best kept secret services of forest ecosystems. Forests, 7(3):60, 2016.

9. V. Dubreuil, D. Arvor, B. M. Funatsu, V. Nédélec, and N. A. d. Mello-Théry. Les changements climatiques en Amazonie, une approche multiscalaire. In D.Mercier, editor, Les impacts spatiaux du changement climatique, pages 247-270. ISTE, 2020.

10. K. Fernandes, A. Giannini, L. Verchot, W. Baethgen, and M. Pinedo-Vasquez. Decadal covariability of Atlantic SSTs and western Amazon dry-season hydroclimate in observations and CMIP5 simulations. Geophysical Research Letters, 42(16):6793-6801, 2015.

11. A. F. Filippov. Differential equations with discontinuous righthand sides: control systems, volume 18. Springer Science \& Business Media, 2013.

12. M. D. Flannigan, B. J. Stocks, and B. M. Wotton. Climate change and forest fires. Science of the total environment, 262(3):221-229, 2000.

13. N. Gillett, A. Weaver, F. Zwiers, and M. Flannigan. Detecting the effect of climate change on Canadian forest fires. Geophysical Research Letters, 31(18), 2004.

14. A. J. Hansen, P. Burns, J. Ervin, S. J. Goetz, M. Hansen, O. Venter, J. E. Watson, P. A. Jantz, A. L. Virnig, K. Barnett, et al. A policy-driven framework for conserving the best of earth's remaining moist tropical forests. Nature Ecology $\&$ Evolution, pages $1-8,2020$.

15. F. Hecht, O. Pironneau, A. Le Hyaric, and K. Ohtsuka. Freefem++ manual, 2005.

16. D. Henry. Geometric theory of semilinear parabolic equations, volume 840. Springer, 2006 .

17. R. J. Keenan. Climate change impacts and adaptation in forest management: a review. Annals of Forest Science, 72(2):145-167, 2015.

18. T. Kohyama. Size-structured multi-species model of rain forest trees. Functional Ecology, pages 206-212, 1992.

19. A. Kolobov and E. Y. Frisman. Individual-based model of spatio-temporal dynamics of mixed forest stands. Ecological Complexity, 27:29-39, 2016. 
20. Y. A. Kuznetsov, M. Y. Antonovsky, V. Biktashev, and E. Aponina. A cross-diffusion model of forest boundary dynamics. Journal of Mathematical Biology, 32(3):219-232, 1994.

21. C. Le Huy, T. Tsujikawa, and A. Yagi. Asymptotic behavior of solutions for forest kinematic model. Funkcialaj Ekvacioj, 49(3):427-449, 2006.

22. C. Le Huy, T. Tsujikawa, and A. Yagi. Stationary solutions to forest kinematic model. Glasgow Mathematical Journal, 51(1):1-17, 2009.

23. P. Magal and Z. Zhang. Competition for light in forest population dynamics: From computer simulator to mathematical model. Journal of theoretical biology, 419:290304, 2017.

24. C. A. Nobre, G. O. Obregón, J. A. Marengo, R. Fu, and G. Poveda. Characteristics of Amazonian climate: Main features. In Amazonia and Global Change, pages 149-162. American Geophysical Union (AGU), 2009.

25. M. North, S. Stephens, B. Collins, J. Agee, G. Aplet, J. Franklin, and P. Z. Fule. Reform forest fire management. Science, 349(6254):1280-1281, 2015.

26. R. F. Noss. Beyond Kyoto: forest management in a time of rapid climate change. Conservation biology, 15(3):578-590, 2001.

27. M. D. Oyama and C. A. Nobre. A new climate-vegetation equilibrium state for Tropical South America. Geophysical Research Letters, 30(23), 2003.

28. S. W. Running, R. R. Nemani, D. L. Peterson, L. E. Band, D. F. Potts, L. L. Pierce, and M. A. Spanner. Mapping regional forest evapotranspiration and photosynthesis by coupling satellite data with ecosystem simulation. Ecology, 70(4):1090-1101, 1989.

29. E. Schertzer, A. Staver, and S. A. Levin. Implications of the spatial dynamics of fire spread for the bistability of savanna and forest. Journal of Mathematical Biology, 70(12):329-341, 2015.

30. H. R. Schwetlick. Limit sets for multidimensional nonlinear transport equations. Journal of Differential Equations, 179(1):356-368, 2002.

31. T. Shirai, L. Chuan, and A. Yagi. Dynamical system for forest kinematic model under Dirichlet conditions. Scientiae Mathematicae japonicae, 66(2):275-288, 2007.

32. J. Shukla and B. Dubey. Modelling the depletion and conservation of forestry resources: effects of population and pollution. Journal of Mathematical Biology, 36(1):71-94, 1997.

33. H. L. Smith and H. R. Thieme. Dynamical systems and population persistence, volume 118. American Mathematical Soc., 2011.

34. D. L. Spittlehouse and R. B. Stewart. Adaptation to climate change in forest management. Journal of Ecosystems and Management, 4(1), 2004.

35. G. Strang. On the construction and comparison of difference schemes. SIAM journal on numerical analysis, 5(3):506-517, 1968.

36. E. Veldkamp, M. Schmidt, J. S. Powers, and M. D. Corre. Deforestation and reforestation impacts on soils in the tropics. Nature Reviews Earth E Environment, pages 1-16, 2020 .

37. N. Verzelen, N. Picard, and S. Gourlet-Fleury. Approximating spatial interactions in a model of forest dynamics as a means of understanding spatial patterns. Ecological Complexity, 3(3):209-218, 2006.

38. T. Webb. Is vegetation in equilibrium with climate? How to interpret late-Quaternary pollen data. Vegetatio, 67(2):75-91, 1986.

39. K. B. Wilson, P. J. Hanson, P. J. Mulholland, D. D. Baldocchi, and S. D. Wullschleger. A comparison of methods for determining forest evapotranspiration and its components: sap-flow, soil water budget, eddy covariance and catchment water balance. Agricultural and forest Meteorology, 106(2):153-168, 2001.

40. A. Yagi. Abstract parabolic evolution equations and their applications. Springer Science \& Business Media, 2009

41. V. Yatat, P. Couteron, J. J. Tewa, S. Bowong, and Y. Dumont. An impulsive modelling framework of fire occurrence in a size-structured model of tree-grass interactions for savanna ecosystems. Journal of Mathematical Biology, 74(6):1425-1482, 2017.

42. R. Yousefpour, B. You, and M. Hanewinkel. Simulation of extreme storm effects on regional forest soil carbon stock. Ecological Modelling, 399:39-53, 2019.

43. D. C. Zemp, C.-F. Schleussner, H. M. Barbosa, M. Hirota, V. Montade, G. Sampaio, A. Staal, L. Wang-Erlandsson, and A. Rammig. Self-amplified Amazon forest loss due to vegetation-atmosphere feedbacks. Nature communications, 8(1):1-10, 2017. 
44. Z. Zheng, W. Huang, S. Li, and Y. Zeng. Forest fire spread simulating model using cellular automaton with extreme learning machine. Ecological Modelling, 348:33-43, 2017.

45. G. Zhou, C. Meng, P. Jiang, and Q. Xu. Review of carbon fixation in bamboo forests in China. The botanical review, 77(3):262, 2011.

\section{Appendix}

In this appendix, we present the proofs of Theorems 1, 2, 3, and we briefly show numerical results which underpin the approximation of the non-stationary advection equation (1) by the stationary advection equation (2).

\subsection{Proof of Theorem 1}

The proof of Theorem 1 is divided into three steps. First, we use the method of characteristics to solve the stationary equation (19), so as to prove that the operator $\psi$ is well defined. Since $v \in L^{\infty}(\Omega)$ may be discontinuous, we use the theory of ordinary differential equations of Carathéodory. In the second step, we prove that the operator $\psi$ is continuous, using an integral Gronwall lemma. Finally, in the third step, we prove that the operator $\psi$ is differentiable. We remark that its derivative is uniquely determined as the solution of an ODE.

First step. Let $\left\{\xi\left(x_{0}, s\right)\right\}_{0 \leq s \leq S\left(x_{0}\right)}$ denote one characteristic line of the advection field $\boldsymbol{a}$, starting at $x_{0} \in \Gamma$ and ending at $\xi\left(x_{0}, S\left(x_{0}\right)\right) \in \partial \Omega \backslash \Gamma$. We introduce the function $\tilde{\rho}$ defined along the latter characteristic line by $\tilde{\rho}(s)=\rho \circ \xi\left(x_{0}, s\right)$, for $0 \leq s \leq S\left(x_{0}\right)$, with the lightened notation (18). Then it is seen that $\tilde{\rho}$ satisfies the following ODE:

$$
\left\{\begin{array}{l}
\frac{d \tilde{\rho}}{d s}+\sigma \tilde{\rho}=\varphi(\tilde{\rho}) \tilde{v}, \quad 0<s \leq S\left(x_{0}\right) \\
\tilde{\rho}(0)=m\left(x_{0}\right) .
\end{array}\right.
$$

Now we introduce the function $\theta$ defined on $\left[0, S\left(x_{0}\right)\right]$ by $\theta(s)=\tilde{\rho}(s) e^{\sigma s}$. We easily prove that $\theta$ satisfies the following ODE:

$$
\left\{\begin{array}{l}
\frac{d \theta}{d s}=g(s, \theta), \quad 0<s \leq S\left(x_{0}\right), \\
\theta(0)=m\left(x_{0}\right)
\end{array}\right.
$$

where $g$ is given by

$$
g(s, \theta)=\varphi\left(\theta e^{-\sigma s}\right) e^{\sigma s} \tilde{v}(s) .
$$

Since $v \in L^{\infty}(\Omega)$, the function $g$ is continuous in its second variable $\theta$ but discontinuous in its first variable $s$. For that reason, we solve equation (44) as an ODE of Carathéodory type (see e.g. [11], Chapter 1). We directly verify the following properties: 
- the function $g(s, \theta)$ is defined in $\left[0, S\left(x_{0}\right)\right] \times \mathbb{R}$, continuous in $\theta$ for almost every $s$;

- for all $\theta$, the function $g(\cdot, \theta)$ is measurable;

- $|g(s, \theta)| \leq M(s)$ with $M(s)=\varphi_{0} e^{\sigma s}|\tilde{v}(s)| \in L^{\infty}\left(\left[0, S\left(x_{0}\right)\right]\right)$;

- $\left|g\left(s, \theta_{1}\right)-g\left(s, \theta_{2}\right)\right| \leq L(s)\left|\theta_{1}-\theta_{2}\right|$ with $L(s)=\varphi_{0}|\tilde{v}(s)| \in L^{\infty}\left(\left[0, S\left(x_{0}\right)\right]\right)$.

By virtue of Theorems 1 and 2 in [11], Chapter 1, equation (44) admits a unique local solution, defined on $[0, \bar{s}]$ with $\bar{s}>0$. That solution is given by

$$
\theta(s)=m\left(x_{0}\right)+\int_{0}^{s} g(\tau, \theta(\tau)) d \tau, \quad 0 \leq s \leq \bar{s}
$$

and it is absolutely continuous on each compact interval included in $[0, \bar{s}]$. Next, the non-negativity of $\theta(s)$ follows from Proposition A.17 in [33] (note that the continuity is not necessary in the proof of that proposition). We continue by proving that $\theta$ is global in $\left[0, S\left(x_{0}\right)\right]$. This simply follows from the differential inequality

$$
\frac{d \theta}{d s} \leq \varphi_{0}\|v\|_{\infty} \theta, \quad s>0
$$

which leads to

$$
\theta(s) \leq m\left(x_{0}\right) e^{\varphi_{0}\|v\|_{\infty} S\left(x_{0}\right)}, \quad \forall s \in[0, \bar{s}]
$$

Thus we have $\bar{s}=S\left(x_{0}\right)$.

Now we are brought back to equation (43), which is solved by setting

$$
\tilde{\rho}(s)=\theta(s) e^{-\sigma s}, \quad s \in\left[0, S\left(x_{0}\right)\right] .
$$

As constructed, $\tilde{\rho}$ is absolutely continuous on each compact interval included in $\left[0, S\left(x_{0}\right)\right]$, and uniquely determined by the representation formula

$$
\tilde{\rho}(s)=m\left(x_{0}\right) e^{-\sigma s}+\int_{0}^{s} \varphi(\tilde{\rho}(\tau)) \tilde{v}(\tau) e^{-\sigma(s-\tau)} d \tau, \quad s \in\left[0, S\left(x_{0}\right)\right],
$$

which is the lightened expression for equation (21). Since equation (43) has been solved along each characteristic line of the advection field $\boldsymbol{a}$, we finally solve equation (19) in $\Omega$ by setting

$$
\rho(x)=\tilde{\rho}\left(\zeta_{1}(x), \zeta_{2}(x)\right), \quad x \in \Omega,
$$

with the notation (18), where $\zeta_{1}$ and $\zeta_{2}$ are defined by (17). As constructed, $\rho$ is continuous and non-negative along the characteristic lines of the advection field $\boldsymbol{a}$. Furthermore, the principle of continuity of the solution of equation (43) with respect to a variation of the initial condition (see Theorem 6 in [11], Chapter 1) and the continuity of the advection field $\boldsymbol{a}$ (see equation (15)) guaranty that $\rho$ is continuous in $\bar{\Omega}$, thus belongs to $L_{+}^{\infty}(\Omega)$. We have proved that the operator $\psi$ is well defined. 
Second step. Let $v, h \in L_{+}^{\infty}(\Omega)$. We set $\rho=\psi(v)$ and $\rho^{h}=\psi(v+h)$. By virtue of the representation formula (21), we have:

$$
\begin{aligned}
& |\psi(v+h)(x)-\psi(v)(x)| \\
& =\left|\int_{0}^{s} \varphi\left(\tilde{\rho}^{h}(\tau)\right)(\tilde{v}+\tilde{h})(\tau) e^{-\sigma(s-\tau)} d \tau-\int_{0}^{s} \varphi(\tilde{\rho}(\tau)) \tilde{v}(\tau) e^{-\sigma(s-\tau)} d \tau\right| \\
& =\left|\int_{0}^{s}\left[\varphi\left(\tilde{\rho}^{h}(\tau)\right)-\varphi(\tilde{\rho}(\tau))\right] \tilde{v}(\tau) e^{-\sigma(s-\tau)} d \tau+\int_{0}^{s} \varphi\left(\tilde{\rho}^{h}(\tau)\right) \tilde{h}(\tau) e^{-\sigma(s-\tau)} d \tau\right| \\
& \leq\|v\|_{\infty} \int_{0}^{s}\left|\varphi\left(\tilde{\rho}^{h}(\tau)\right)-\varphi(\tilde{\rho}(\tau))\right| d \tau+\|h\|_{\infty} \varphi_{0} \int_{0}^{s} e^{-\sigma(s-\tau)} d \tau \\
& \leq\|v\|_{\infty} \varphi_{0} \int_{0}^{s}\left|\tilde{\rho}^{h}(\tau)-\tilde{\rho}(\tau)\right| d \tau+\|h\|_{\infty} \frac{\varphi_{0}}{\sigma},
\end{aligned}
$$

where we have used properties of the function $\varphi$ given in (13). Now we introduce the function $p$ defined by

$$
p(s)=\left|\tilde{\rho}^{h}(s)-\tilde{\rho}(s)\right|, \quad s \geq 0 .
$$

We have proved above that

$$
p(s) \leq\|v\|_{\infty} \varphi_{0} \int_{0}^{s} p(\tau) d \tau+\|h\|_{\infty} \frac{\varphi_{0}}{\sigma} .
$$

By virtue on the integral Gronwall lemma, we obtain

$$
p(s) \leq\|h\|_{\infty} \frac{\varphi_{0}}{\sigma} e^{\|v\|_{\infty} \varphi_{0} s} \leq\|h\|_{\infty} \frac{\varphi_{0}}{\sigma} e^{\|v\|_{\infty} \varphi_{0} \bar{S}},
$$

where we have used the uniform bound (16). We then obtain

$$
\|\psi(v+h)-\psi(v)\|_{\infty} \leq\|h\|_{\infty} \times \frac{\varphi_{0}}{\sigma} e^{\varphi_{0}\|v\|_{\infty} \bar{S}},
$$

which proves the continuity of the operator $\psi$.

Third step. Finally, let us introduce the operator $L_{v}$ uniquely defined for all $v \in L_{+}^{\infty}(\Omega)$ by the integral formula

$$
L_{v} \tilde{h}(s)=\int_{0}^{s} \varphi(\tilde{\rho}(\tau)) \tilde{h}(\tau) e^{-\sigma(s-\tau)} d \tau+\int_{0}^{s} \varphi^{\prime}(\tilde{\rho}(\tau)) \tilde{v}(\tau) L_{v} \tilde{h}(\tau) e^{\sigma(s-\tau)} d \tau,
$$

for all $h \in L_{+}^{\infty}(\Omega)$, where $\rho=\psi(v)$. As constructed, the operator $L_{v}$ is obviously linear. Now, let $v, h \in L_{+}^{\infty}(\Omega)$. We set again $\rho=\psi(v)$ and $\rho^{h}=\psi(v+h)$, and we introduce the function $p$ defined by

$$
p(s)=\left|\tilde{\rho}^{h}(s)-\tilde{\rho}(s)-L_{v} h(s)\right|, \quad s \geq 0 .
$$

We use the continuity and differentiability of the function $\varphi$ in order to write:

$$
\begin{aligned}
& \varphi\left(\tilde{\rho}^{h}\right)=\varphi(\tilde{\rho})+o(1) \text { as }\|h\|_{\infty} \rightarrow 0, \\
& \varphi\left(\tilde{\rho}^{h}\right)=\varphi(\tilde{\rho})+\varphi^{\prime}(\tilde{\rho})\left(\tilde{\rho}^{h}-\tilde{\rho}\right)+o\left(\|h\|_{\infty}\right) \text { as }\|h\|_{\infty} \rightarrow 0,
\end{aligned}
$$


which leads to, after basic computations:

$$
p(s) \leq\|v\|_{\infty} \int_{0}^{s} p(\tau) d \tau+o\left(\|h\|_{\infty}\right) \times \frac{\|v\|_{\infty}+1}{\sigma}, \quad s \geq 0 .
$$

Using the integral Gronwall lemma again and equation (16) leads to

$$
\left|\tilde{\rho}^{h}(s)-\tilde{\rho}(s)-L_{v} h(s)\right| \leq o\left(\|h\|_{\infty}\right) \times \frac{\|v\|_{\infty}+1}{\sigma} e^{\|v\|_{\infty} \bar{S}} .
$$

Furthermore, we easily observe that

$$
\left|L_{v} \tilde{h}(s)\right| \leq\|v\|_{\infty} \int_{0}^{s}\left|L_{v} \tilde{h}(\tau)\right|+\frac{\varphi_{0}\|h\|_{\infty}}{\sigma} .
$$

Using a third time the integral Gronwall lemma leads to

$$
\left|L_{v} \tilde{h}(s)\right| \leq \frac{\varphi_{0}\|h\|_{\infty}}{\sigma} e^{\|v\|_{\infty} \bar{S}}
$$

In this way, we have proved that $\psi$ is differentiable in $L^{\infty}(\Omega)$. Its derivative is given by

$$
D \psi(v) h=L_{v} h,
$$

for all $v, h \in L_{+}^{\infty}(\Omega)$ and satisfies $(24)$.

\subsection{Proof of Theorem 2}

By virtue of Theorem 4.4 in [40], it suffices to prove an estimation of the type

$$
\begin{aligned}
& \|F(U)-F(\tilde{U})\|_{X} \\
& \quad \leq k\left(\|U\|_{X}+\|\tilde{U}\|_{X}\right)\left[\left\|A^{\eta}(U-\tilde{U})\right\|_{X}+\left(\|U\|_{X}+\|\tilde{U}\|_{X}\right)\|U-\tilde{U}\|_{X}\right],
\end{aligned}
$$

for each $U, \tilde{U}$ in $\mathcal{D}\left(A^{\eta}\right)$, where $k$ is an increasing function defined in $\mathbb{R}^{+}$with values in $\mathbb{R}^{+}$. Thus we consider $U=(u, v, w)^{T}, \tilde{U}=(\tilde{u}, \tilde{v}, \tilde{w})^{T}$ in $\mathcal{D}\left(A^{\eta}\right)$. We begin by estimating the norm of the difference $\left\|F_{1}(U)-F_{1}(\tilde{U})\right\|_{\infty}$. To that aim, we write:

$$
\begin{aligned}
\left\|F_{1}(U)-F_{1}(\tilde{U})\right\|_{\infty} & \leq \beta \delta\|w-\tilde{w}\|_{\infty} \\
& +\left\|\gamma_{0}(v) u-\gamma_{0}(\tilde{v}) \tilde{u}\right\|_{\infty} \\
& +\|\mu(\psi(v)) u-\mu(\psi(\tilde{v})) \tilde{u}\|_{\infty} .
\end{aligned}
$$

Next, we use the expression of $\gamma_{0}(v)$ given in $(9)$ :

$\left\|\gamma_{0}(v) u-\gamma_{0}(\tilde{v}) \tilde{u}\right\|_{\infty} \leq a\left\|v^{2} u-\tilde{v}^{2} \tilde{u}\right\|_{\infty}+2 a b\|v u-\tilde{v} \tilde{u}\|_{\infty}+\left(a b^{2}+c\right)\|u-\tilde{u}\|_{\infty}$.

But we have

$$
\begin{aligned}
\|v u-\tilde{v} \tilde{u}\|_{\infty} & \leq\|v u-v \tilde{u}\|_{\infty}+\|v \tilde{u}-\tilde{v} \tilde{u}\|_{\infty} \\
& \leq\|v\|_{\infty}\|u-\tilde{u}\|_{\infty}+\|\tilde{u}\|_{\infty}\|v-\tilde{v}\|_{\infty} \\
& \leq\left(\|U\|_{X}+\|\tilde{U}\|_{X}\right)\|U-\tilde{U}\|_{X} .
\end{aligned}
$$


Similarly, we have:

$$
\begin{aligned}
\left\|v^{2} u-\tilde{v}^{2} \tilde{u}\right\|_{\infty} & \leq\|v\|_{\infty}^{2}\|u-\tilde{u}\|_{\infty}+\|\tilde{u}\|_{\infty}\left(\|v\|_{\infty}+\|\tilde{v}\|_{\infty}\right)\|v-\tilde{v}\|_{\infty} \\
& \leq\left(\|U\|_{X}^{2}+\|\tilde{U}\|_{X}^{2}\right)\|U-\tilde{U}\|_{X} .
\end{aligned}
$$

Afterwards, we write:

$$
\begin{aligned}
\|\mu(\psi(v)) u-\mu(\psi(\tilde{v})) \tilde{u}\|_{\infty} & \leq\|\mu(\psi(v)) u-\mu(\psi(v)) \tilde{u}\|_{\infty} \\
& +\|\mu(\psi(v)) \tilde{u}-\mu(\psi(\tilde{v})) \tilde{u}\|_{\infty} \\
& \leq \mu_{0}\|u-\tilde{u}\|_{\infty}+\|\tilde{u}\|_{\infty} \times \mu_{0}\|\psi(v)-\psi(\tilde{v})\|_{\infty} \\
& \leq \mu_{0}\|u-\tilde{u}\|_{\infty}+\|\tilde{u}\|_{\infty} \times\|v-\tilde{v}\|_{\infty} \frac{\mu_{0} \varphi_{0}}{\sigma} e^{\varphi_{0}\|v\|_{\infty} \bar{S}},
\end{aligned}
$$

where we have used estimation (22) at the last step. Combining the above inequalities leads to

$$
\left\|F_{1}(U)-F_{1}(\tilde{U})\right\|_{\infty} \leq k_{1}\left(\|U\|_{X}+\|\tilde{U}\|_{X}\right)\left(\|U\|_{X}+\|\tilde{U}\|_{X}\right)\|U-\tilde{U}\|_{X},
$$

where $k_{1}$ is a continuous increasing function. We estimate $\left\|F_{2}(U)-F_{2}(\tilde{U})\right\|_{\infty}$ analogously.

It remains to estimate the norm of the difference $\left\|F_{3}(U)-F_{3}(\tilde{U})\right\|_{2}$. We write:

$$
\begin{aligned}
& \|\alpha(\psi(v)) v-\alpha(\psi(\tilde{v})) \tilde{v}\|_{2} \\
& \quad \leq\|\alpha(\psi(v)) v-\alpha(\psi(\tilde{v})) v\|_{2}+\|\alpha(\psi(\tilde{v})) v-\alpha(\psi(\tilde{v})) \tilde{v}\|_{2} \\
& \quad \leq\|v\|_{\infty}\|\alpha(\psi(v))-\alpha(\psi(\tilde{v}))\|_{2}+\|\alpha(\psi(\tilde{v}))\|_{\infty}\|v-\tilde{v}\|_{2} \\
& \quad \leq \alpha_{0}\|v\|_{\infty}\|\psi(v)-\psi(\tilde{v})\|_{2}+\alpha_{0}\|v-\tilde{v}\|_{2},
\end{aligned}
$$

where we have used the properties of the function $\alpha$ given in (13). Now we use the embedding $L^{\infty}(\Omega) \subset L^{2}(\Omega)$ and estimation (22), which leads to

$$
\|\alpha(\psi(v)) v-\alpha(\psi(\tilde{v})) \tilde{v}\|_{2} \leq C\left[\|v\|_{\infty}\|v-\tilde{v}\|_{\infty} \frac{\varphi_{0}}{\sigma} e^{\varphi_{0}\|v\|_{\infty} \bar{S}}+\|v-\tilde{v}\|_{2}\right],
$$

where $C$ denotes a positive constant. Since $\|v-\tilde{v}\|_{2} \leq C\left\|A^{\eta}(U-\tilde{U})\right\|_{X}$, we obtain:

$$
\begin{aligned}
& \|\alpha(\psi(v)) v-\alpha(\psi(\tilde{v})) \tilde{v}\|_{2} \\
& \leq k_{3}\left(\|U\|_{X}+\|\tilde{U}\|_{X}\right)\left[\left\|A^{\eta}(U-\tilde{U})\right\|_{X}+\left(\|U\|_{X}+\|\tilde{U}\|_{X}\right)\|U-\tilde{U}\|_{X}\right],
\end{aligned}
$$

where $k_{3}$ denotes a continuous increasing function. Combining the above estimates leads to the desired conclusion. 


\subsection{Proof of Theorem 3}

If $s(0)>0$, the spectral condition $\operatorname{Spec}(-\bar{A}) \subset\{z \in \mathbb{C}: \Re(z) \leq-\varepsilon\}$ for some $\varepsilon>0$ follows directly from Lemma 1 and the above properties of the function $s$. Next, the local stability of the stationary state $(\bar{\rho}(x), 0,0,0)$ is guaranteed by the results of [40] (Section 6.2).

If $s(0)<0$, if follows again from Lemma 1 that $\operatorname{Spec}(-\bar{A})$ contains a real positive value. To prove that the stationary state $(\bar{\rho}(x), 0,0,0)$ is unstable, by virtue of Corollary 5.1.6 in [16], it suffices to check that

$$
\left\|F(\bar{U}+z)-F(\bar{U})-F^{\prime}(\bar{U}) z\right\|_{X}=O\left(\|z\|_{X}^{p}\right),
$$

for $z$ in a neighborhood of 0 and $p>1$. To this end, we easily compute

$$
F(\bar{U}+z)-F(\bar{U})-F^{\prime}(\bar{U}) z=\left(\begin{array}{c}
-\gamma\left(z_{2}, \psi\left(z_{2}\right)\right) z_{1}+\gamma(0, \psi(0)) z_{1} \\
-h\left(\psi\left(z_{2}\right)\right) z_{2}+h(\psi(0)) z_{2} \\
\alpha\left(\psi\left(z_{2}\right)\right) z_{2}-\alpha(\psi(0)) z_{2}
\end{array}\right)
$$

for all $z=\left(z_{1}, z_{2}, z_{3}\right)^{T} \in X$. Recall that $X=L^{\infty}(\Omega) \times L^{\infty}(\Omega) \times L^{2}(\Omega)$. We have

$$
\begin{aligned}
\left\|\gamma\left(z_{2}, \psi\left(z_{2}\right)\right) z_{1}-\gamma(0, \psi(0)) z_{1}\right\|_{\infty} & \leq\left\|\left[a\left(z_{2}-b\right)^{2}+c-\left(a b^{2}+c\right)\right] z_{1}\right\|_{\infty} \\
& +\left\|\left[\mu\left(\psi\left(z_{2}\right)\right)-\mu(\psi(0))\right] z_{1}\right\|_{\infty} .
\end{aligned}
$$

First, it is easily seen that

$$
\left\|\left[a\left(z_{2}-b\right)^{2}+c-\left(a b^{2}+c\right)\right] z_{1}\right\|_{\infty}=O\left(\|z\|_{X}^{2}\right),
$$

as $\|z\|_{X}$ tends to 0 . Furthermore, since the function $\mu$ and the operator $\psi$ are differentiable, we have

$$
\left\|\mu\left(\psi\left(z_{2}\right)\right)-\mu(\psi(0))\right\|_{\infty}=O\left(\|z\|_{X}\right),
$$

from which it follows that

$$
\left\|\left[\mu\left(\psi\left(z_{2}\right)\right)-\mu(\psi(0))\right] z_{1}\right\|_{\infty}=O\left(\|z\|_{X}^{2}\right),
$$

as $\|z\|_{X}$ tends to 0 . We obtain

$$
\left\|\gamma\left(z_{2}, \psi\left(z_{2}\right)\right) z_{1}-\gamma(0, \psi(0)) z_{1}\right\|_{\infty}=O\left(\|z\|_{X}^{2}\right)
$$

as $\|z\|_{X}$ tends to 0 . Since the functions $h$ and $\alpha$ are similarly differentiable, it is shown analogously that

$$
\left\|-h\left(\psi\left(z_{2}\right)\right) z_{2}+h(\psi(0)) z_{2}\right\|_{\infty}=O\left(\|z\|_{X}^{2}\right)
$$

and

$$
\left\|\alpha\left(\psi\left(z_{2}\right)\right) z_{2}-\alpha(\psi(0)) z_{2}\right\|_{2}=O\left(\|z\|_{X}^{2}\right),
$$

which proves (45) with $p=2$. The proof is complete. 
7.4 Approximation of the non-stationary advection equation (1) by the stationary advection equation (2)

In order to support the approximation of the non-stationary advection equation (1) by the stationary advection equation (2), we compute numerically the solution of the following system with stationary advection

$$
\left\{\begin{aligned}
\boldsymbol{a} \cdot \nabla \rho & =-\sigma \rho+\varphi(\rho) v \\
\frac{\partial u}{\partial t} & =\beta \delta w-\gamma(v, \rho) u-f u \\
\frac{\partial v}{\partial t} & =f u-h(\rho) v \\
\frac{\partial w}{\partial t} & =d \Delta w-\beta w+\alpha(\rho) v
\end{aligned}\right.
$$

and in parallel, we compute the solution of the following system with nonstationary advection

$$
\left\{\begin{aligned}
\frac{\partial \tilde{\rho}}{\partial t}+\boldsymbol{a} \cdot \nabla \tilde{\rho} & =-\sigma \tilde{\rho}+\varphi(\tilde{\rho}) \tilde{v} \\
\frac{\partial \tilde{u}}{\partial t} & =\beta \delta \tilde{w}-\gamma(\tilde{v}, \tilde{\rho}) u-f \tilde{u} \\
\frac{\partial \tilde{v}}{\partial t} & =f \tilde{u}-h(\tilde{\rho}) \tilde{v} \\
\frac{\partial \tilde{w}}{\partial t} & =d \Delta \tilde{w}-\beta \tilde{w}+\alpha(\tilde{\rho}) \tilde{v}
\end{aligned}\right.
$$

with the same initial conditions and the same parameters. In both cases, the solutions are attracted to the stationary heterogeneous solution $(\bar{\rho}(x), 0,0,0)$ given in Proposition 3, as depicted in the following figures. 

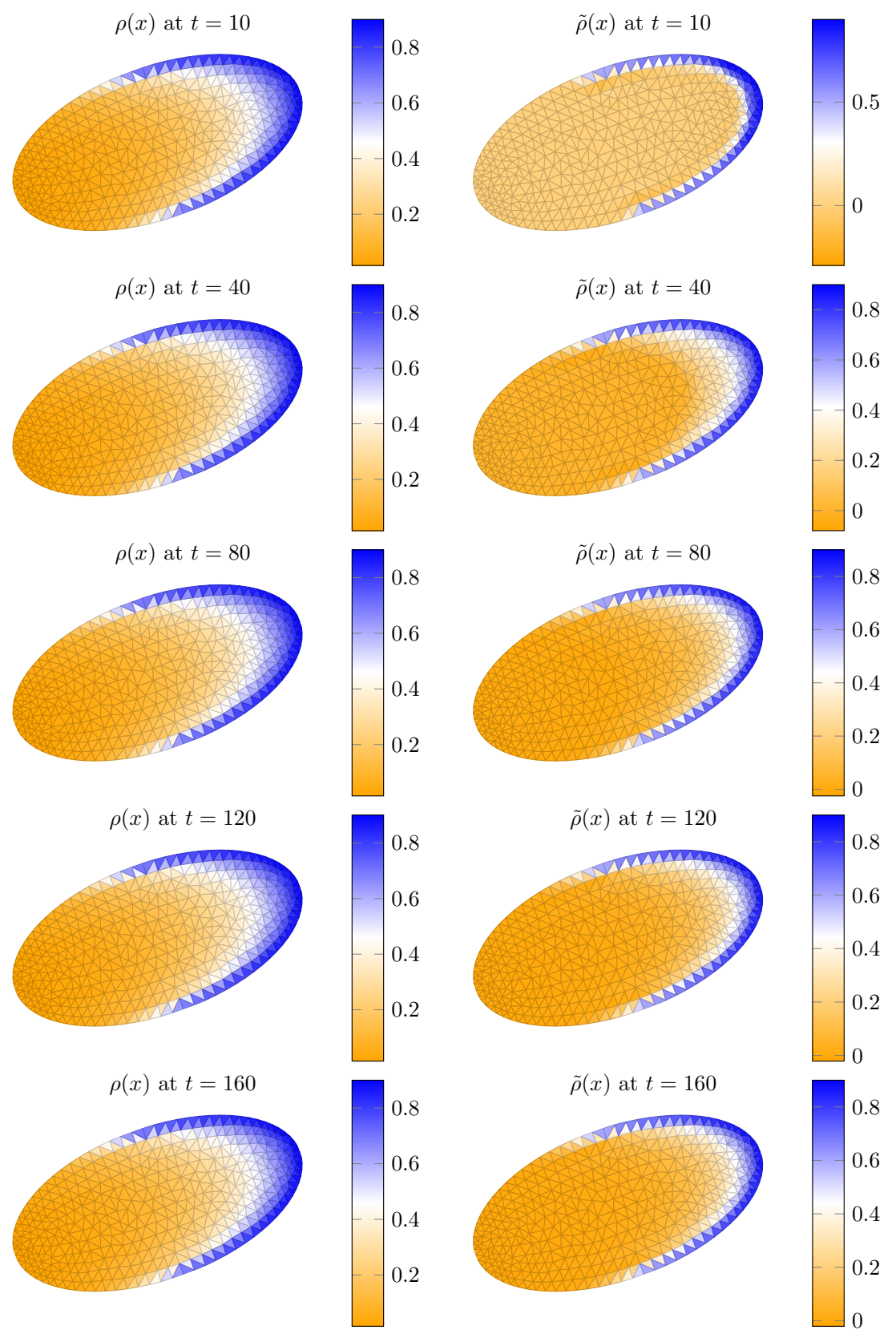

Fig. 12 Numerical results for $\rho(x)$ and $\tilde{\rho}(x)$. Left: stationary advection. Right: nonstationary advection. 


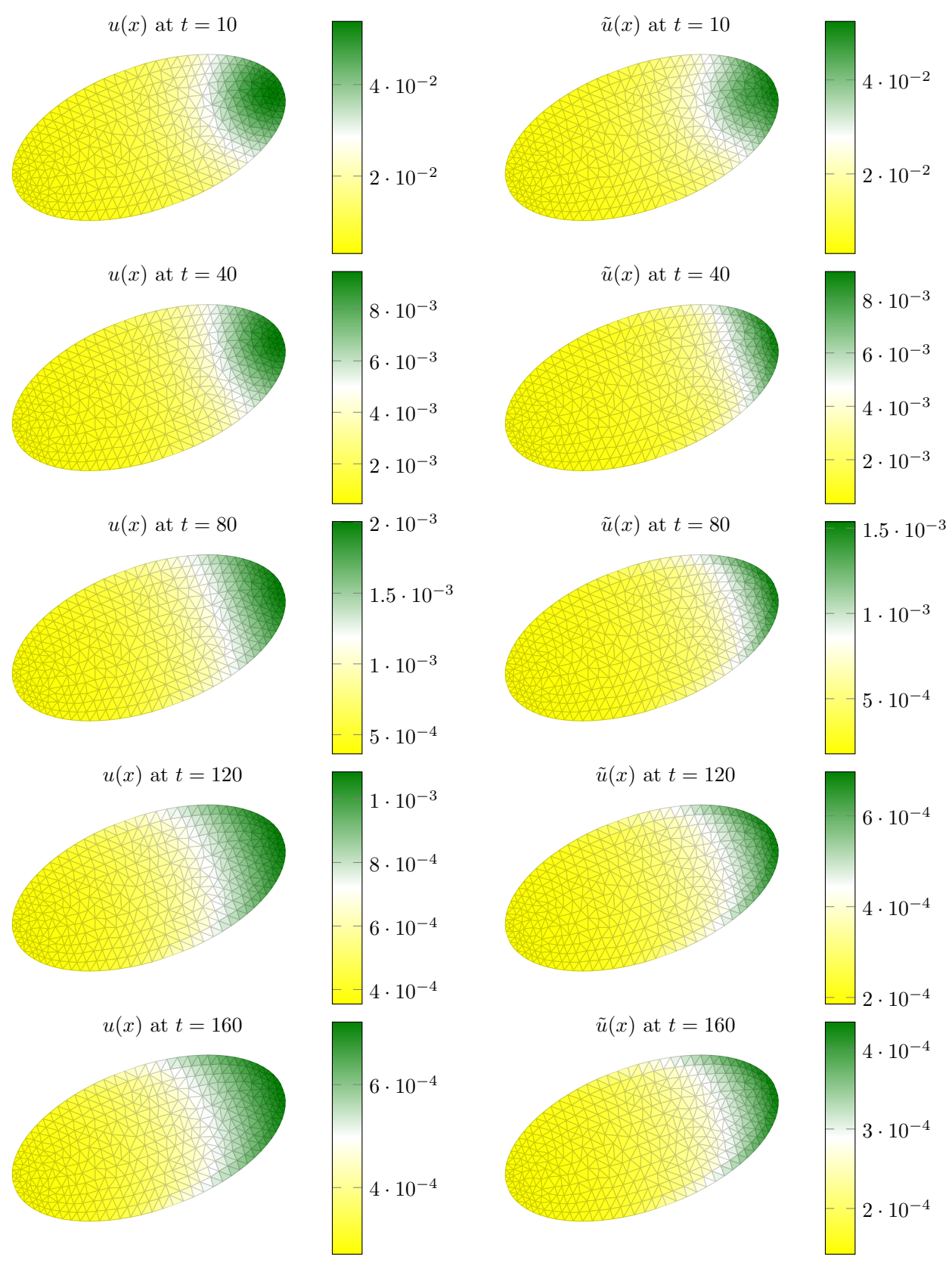

Fig. 13 Numerical results for $u(x)$ and $\tilde{u}(x)$. Left: stationary advection. Right: nonstationary advection. 

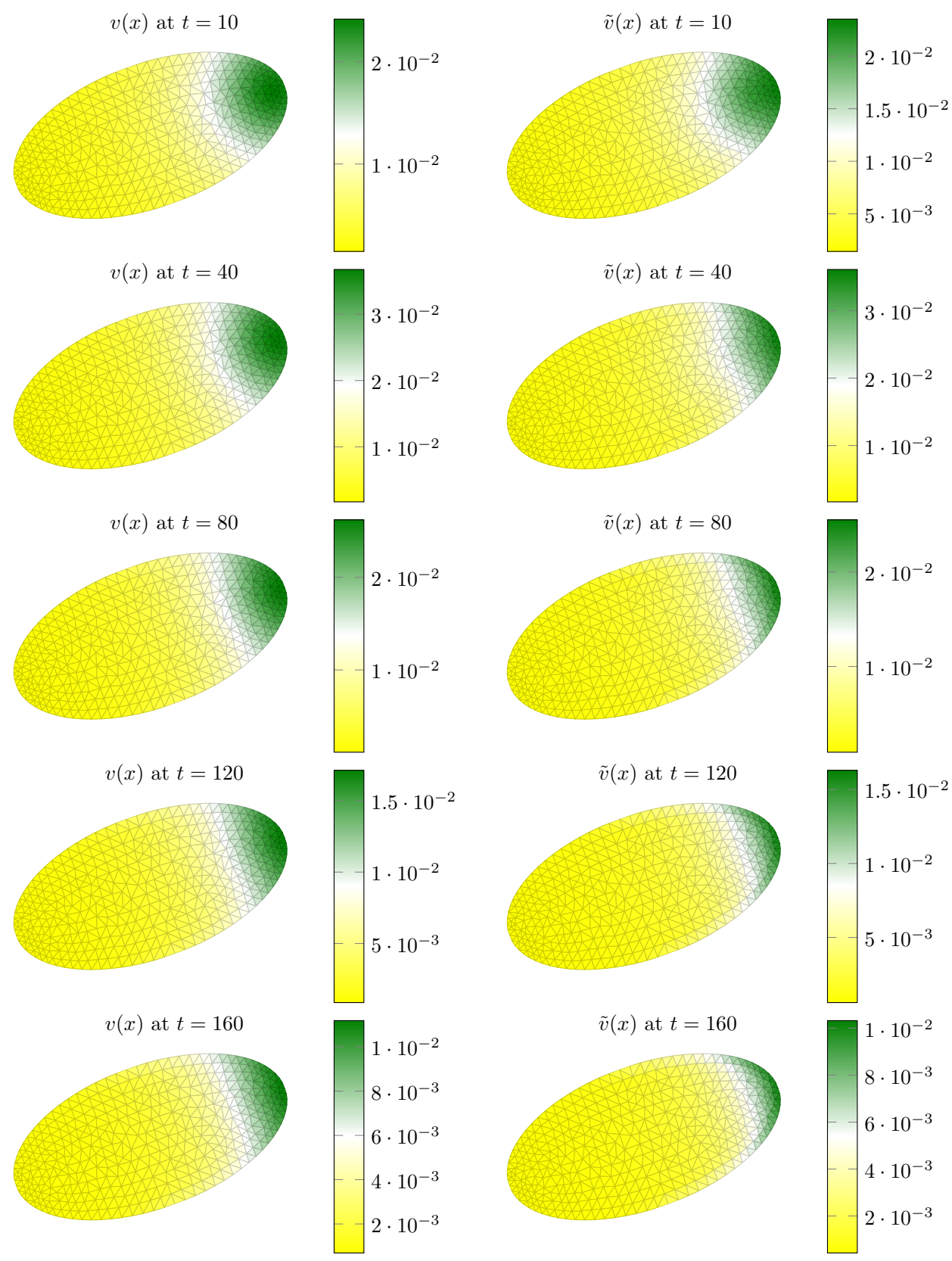

Fig. 14 Numerical results for $v(x)$ and $\tilde{v}(x)$. Left: stationary advection. Right: nonstationary advection. 

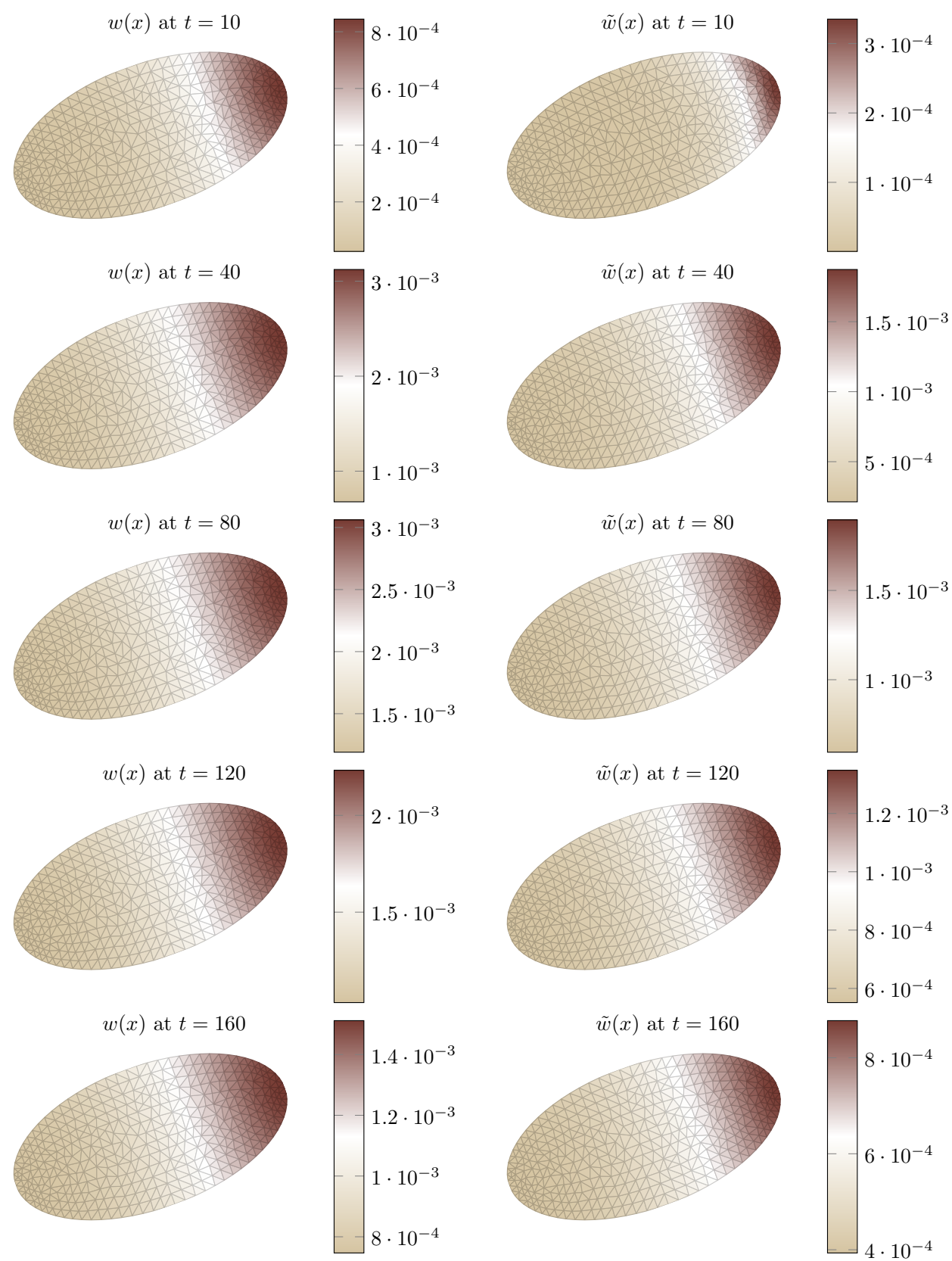

Fig. 15 Numerical results for $w(x)$ and $\tilde{w}(x)$. Left: stationary advection. Right: nonstationary advection. 